\title{
A Randomness Perspective on Intelligence Processes
}

\author{
Inhan Kang ${ }^{1}$, Paul De Boeck ${ }^{1}$, Ivailo Partchev ${ }^{2}$ \\ ${ }^{1}$ Department of Psychology, The Ohio State University, 1827 Neil Avenue, Columbus, OH 43210, United \\ States \\ ${ }^{2}$ Cito, Amsterdamseweg 13, 6814 CM Arnhem, Netherlands
}

Address correspondence:

Paul De Boeck

deboeck.2@osu.edu

The Ohio State University

240K Lazenby Hall

1827 Neil Avenue

Columbus, Ohio 43210 


\begin{abstract}
We study intelligence processes using a diffusion IRT model with random variability in cognitive model parameters: variability in drift rate (the trend of information accumulation toward a correct or incorrect response) and variability in starting point (from where the information accumulation starts). The random variation concerns randomness across person-item pairs and cannot be accounted for by individual and inter-item differences. Interestingly, the models explain the conditional dependencies between response accuracy and response time that are found in previous studies on cognitive ability tests, leading us to the formulation of a randomness perspective on intelligence processes. For an empirical test, we have analyzed verbal analogies data and matrix reasoning data using diffusion IRT models with different variability assumptions. The results indicate that 1) models with random variability fit better than models without, with implications for the conditional dependencies in both types of tasks; 2) for verbal analogies, random variation in drift rate seems to exist, which can be explained by person-by-item word knowledge differences; and 3) for both types of tasks, the starting point variation was also established, in line with the inductive nature of the tasks, requiring a sequential hypothesis testing process. Finally, the correlation of individual differences in drift rate and SAT suggests a meta-strategic choice of respondents to focus on accuracy rather than speed when they have a higher cognitive capacity and when the task is one for which investing in time pays off. This seems primarily the case for matrix reasoning and less so for verbal analogies.
\end{abstract}

Keywords: Conditional dependency, Intelligence processes, Randomness, Diffusion IRT model, Verbal analogies, Matrix reasoning 


\section{Introduction}

The study of intelligence in terms of its processes is a core interest of intelligence research. Knowing the processes helps us understand what intelligence is. Early in the history of the study of intelligence, Spearman (1923) has formulated a process theory, with three processes, which in terms of solving analogies are understanding the problem ("apprehension of experience"), inferring relationships ("eduction of relations"), and application of relationships ("eduction of correlates"). It is therefore not surprising that R. J. Sternberg (1977b, 1977a) started his revolutionary work on cognitive processes underlying intelligence with models for the processes respondents go through when solving analogy problems. An important aspect of Sternberg's work was that response accuracy and response times (RTs) were modeled (although not in a joint model for both) and the data were item-level data. His work was in line with research paradigms from experimental cognitive psychology (e.g., S. Sternberg, 1969). From the psychometric side of the spectrum, Embretson (Whitely, 1976, 1977), has focused on an item-level process analysis of accuracy data with models inspired by item response theory (IRT). In neither of these research lines, a joint modeling approach was used for response accuracy and RTs. Unfortunately, these promising process-related approaches to the study of intelligence have not led to a general breakthrough, although some promising ingredients were used: response accuracy, RTs, an item-level approach (in contrast with global test scores), and explicit and specific process models (instead of general correlation- and covariance-based type of analysis such as factor analysis of test scores). Although factor models are not explicit process models, they can still be useful for process research. For example, R. J. Sternberg (1977a)'s process modeling approach can be reformulated in terms of factor models. However, to capture processes, the items need to be designed to make that possible.

Foregoing many other interesting initiatives, a rather recent process-based approach is to use the diffusion model (Ratcliff, 1978; Ratcliff \& McKoon, 2008) in the study of intelligence processes (Frischkorn \& Schubert, 2018; Lerche et al., 2020; McKoon \& Ratcliff, 2012; 
Ratcliff, Schmiedek, \& McKoon, 2008; Ratcliff, Thapar, \& McKoon, 2009, 2011: Schmiedek, Oberauer, Wilhelm, Süß, \& Wittmann, 2007; Schulz-Zhecheva, Voelkle, Beauducel, Biscaldi, \& Klein, 2016; Schmitz \& Wilhelm, 2016; Schubert \& Frischkorn, 2020; Schubert, Hagemann, Voss, Schankin, \& Bergmann, 2015). A major difference from the earlier approaches is that, in the diffusion model, there are only a few simple and general processes: a non-differentiated category of nondecision processes, information accumulation, and a decision. The model parameters correspond to different components of the cognitive decision processes: a non-decision time parameter to capture the amount of time for nondecision processes, a drift rate parameter for the efficiency of information processing and evidence accumulation, and a boundary separation parameter for the amount of information required to reach a solution (i.e., the decision threshold). A fourth parameter, which is the starting point, represents the initial bias of the response processes and is often chosen to be halfway the two decision boundaries.

Although the diffusion model has been developed for simple binary choices in perceptual and cognitive decision-making tasks, van der Maas, Molenaar, Maris, Kievit, and Borsboom (2011) have extended and applied the model for more complex cognitive tasks that are used in intelligence tests and other cognitive ability tests (called a 'diffusion IRT model'; see also Molenaar, Tuerlinckx, \& van der Maas, 2015a; Tuerlinckx \& De Boeck, 2005; Tuerlinckx, Molenaar, \& van der Maas, 2016). The diffusion IRT model approach was presented as an alternative to the hierarchical framework of van der Linden (2007). Both the diffusion IRT model and the hierarchical framework are joint models for response accuracy and RT, but the former is based on a process theory and offers a solution for the problem of speed-accuracy trade-off (SAT) through the boundary separation parameter. In line with earlier developments, the diffusion model stems from a cognitive psychology line of thinking with a focus on processes and the hierarchical model stems from a psychometric line of thinking with a focus on the measurement of abilities. From a purely formal and data analysis perspective, the two major dimensions in the hierar- 
chical model, ability and speed, define a two-dimension space of individual differences that also is covered reasonably well by the drift rate and boundary separation parameters of the diffusion model (De Boeck, Chen, \& Davison, 2017; De Boeck \& Jeon, 2019). The relationship is not a one-on-one relationship, since a higher drift rate and a larger boundary separation are both beneficial for the accuracy of responses and a high drift rate and a small boundary separation, lead to faster responses.

van der Linden's hierarchical psychometric model for ability and speed and van der Maas et al.'s diffusion IRT model differ in some important other aspects, they both are reasonable and successful modeling approaches to capture the mainly two-dimensional individual differences in response accuracy and RT data from cognitive ability tests. Apart from its process-based theory, the diffusion model has an interesting necessary feature when applied to more complex cognitive problem-solving tasks instead of the simple choice tasks for which it has been mainly used. The information accumulation process from the simple choice tasks needs to be reconceptualized as a heterogeneous and complex process for more complex tasks. It can hardly be an isomorphic representation of the many ongoing processes and steps within these processes, but it still can be an approximate representation. Isomorphic means that the model is a realistic one-to-one representation of the specific processes, whereas an approximate model preserves a predictive correspondence between input and outputs with an approximate explanation of the ongoing processes (Hammond, 1955; Hoffman, 1960).

The information accumulation process component from the diffusion model for complex cognitive tasks has an interesting and perhaps unexpected feature. Many types of phenomena are based on a complex set and interplay of fine-grained events that can be approached at the system level with a degree of simplicity that would not be possible when looking underneath the system level. It seems impossible to map out the large variety of possible cognitive processes, trials and errors, respondents go through while working on a cognitive task. It would be overly ambitious trying to do so. Interestingly, the 
information accumulation process from the diffusion model is basically a continuous-time random walk process but with its drift rate toward one of the two decision boundaries so that it offers system-level approach to the complexity underneath without having to be specific and detailed about what is underneath. It is comparable with modeling the paths of leaves in Fall based on gravity and the wind direction, instead of all the intricacies of leaves whirling down. We all have experienced the many complex and ever-changing cognitive processes we sometimes go through when making a test. It does not seem possible to capture everything. It is an alternative approach to focus more on system features, which in this case implies moving to an approximate approach for the informational accumulation drift but preserving a certain form of randomness in the way problems are solved.

\subsection{A Randomness Perspective on Intelligence Processes}

While there may be randomness within the component of information accumulation (and its approximate interpretation here) for a respondent working on an item, there also may be randomness across pairs of persons and items. This second type of randomness implies that the process parameters vary in a way that cannot be fully captured by individual differences and item differences in the parameter values. The reason to introduce this additional form of randomness is that it can explain residual dependencies between response accuracy and RT as established for cognitive ability items. The residual dependencies refer to dependencies after ruling out global individual differences and item differences (Bolsinova, De Boeck, \& Tijmstra, 2017; Bolsinova \& Maris, 2016; Bolsinova, Tijmstra, \& Molenaar, 2017, Bolsinova, Tijmstra, Molenaar, \& De Boeck, 2017, Bolsinova \& Molenaar, 2018; Chen, De Boeck, Grady, Yang, \& Waldschmidt, 2018a; De Boeck et al. 2017; De Boeck \& Jeon, 2019; Goldhammer et al., 2014: Goldhammer, Naumann, \& Greiff, 2015; Kang, De Boeck, \& Ratcliff, accepted for publication; Meng, Tao, \& Chang, 2015, Partchev \& De Boeck, 2012; van Rijn \& Ali, 2017; van der Linden \& Glas, 2010; Wang \& 
Xu, 2015). Based on many different studies, these dependencies are a common finding. The diffusion model is an ideal approach to explain these dependencies because its parameters are, as cognitive components of the response processes, at the basis of response accuracy and RT. Thus, the random variation of the parameters beyond their person-wise means and item-wise means (and the corresponding model parameters) would create dependencies that cannot be captured through the usual person and item parameters.

The dependencies can be investigated with conditional accuracy functions (CAF) because they reflect the relationship between response accuracy and RT after controlling for SAT differences between persons and items (as captured through the boundary separation parameters). Bolsinova and Molenaar (2018) and Chen et al. (2018a) have found that the residual dependencies are curvilinear. They start out as positive at the lower end of residual RTs, and they level off and turn negative toward the upper end of residual RTs. In other words, response accuracy first increases and then decreases. It was found by Kang et al. (accepted for publication) that a curvilinear CAF can be explained by a combination of the random variations in drift rate and in starting point implemented in diffusion IRT models. The Ratcliff diffusion model also has variability (across trials of a psychological experiment) in nondecision time. Although adding this additional variability can potentially improve model fit, it was not studied in Kang et al. (accepted for publication) and in the presented study because changes in nondecision time do not do not affect predicted patterns of conditional dependency between responses and RT.

Both these variations make sense from a cognitive process point of view. The variation in drift rate can be expected for two reasons. First, as far as solving a cognitive task relies on previously acquired knowledge, one may expect variation across pairs of persons and items because prior knowledge and its accessibility depends on the specificities of previous experiences. Even though there are global individual differences and item differences, there also may be item-specific individual differences and person-specific item differences, based on individual experience. Second, as far as attention, motivation, con- 
centration, etc., plays a role in the information accumulation, the drift rate may not be perfectly stable for the whole time of going through a test, so that variation of the drift rate may be expected. This variation would imply that even for items without any influence from prior knowledge (e.g., Raven type items compared with vocabulary tasks), the drift rate would vary. The first source of variation cannot play a role if the task is not at least partially knowledge-based, so that in such a case only the second source seems plausible. Interestingly, the data we will use in our study stem from verbal analogies for which vocabulary knowledge may play a role and Raven-like matrix problems for which knowledge does not play a role.

The variation of the starting point is usually interpreted as variability in initial bias (e.g., due to responses to earlier items) of decision processes or variability in initial amount of information (e.g., from prior knowledge). For psychometric tests, this variation can also be interpreted as related to the rule finding process in inductive tasks. Rule finding consists of generating hypothetical rules to be tested as underlying the presented stimuli, so that the set of stimuli can be completed with a response. The initial hypotheses can be interpreted as the starting point. Because the hypothesis generation process is partly a trial-and-error process, one can expect random variation of how close the starting point is to the boundary of a correct or incorrect response. One can be lucky (or unlucky) with early-in-the-process hypotheses on the rule that applies in an inductive reasoning task.

Interestingly, Kang et al. (accepted for publication) showed what the consequences of the random variations in drift rate and in starting point are for the CAFs (Figure 1). For rather easy tasks (the top side of Figure 1] with a positive drift rate $\nu=2$ ), the variation in the starting point creates an initial upward curve (green dotted line) whereas variation in the drift rate creates a downward curve, with slower RTs being associated with a lower accuracy rate (slower is less accurate, red dashed line). The combination of the two leads to a curvilinear shape (two dot-dashed lines). For difficult tasks (the bottom-side of Figure 1 with a negative drift rate $\nu=-2$ ), the curvilinearity is the mirror image, as shown 


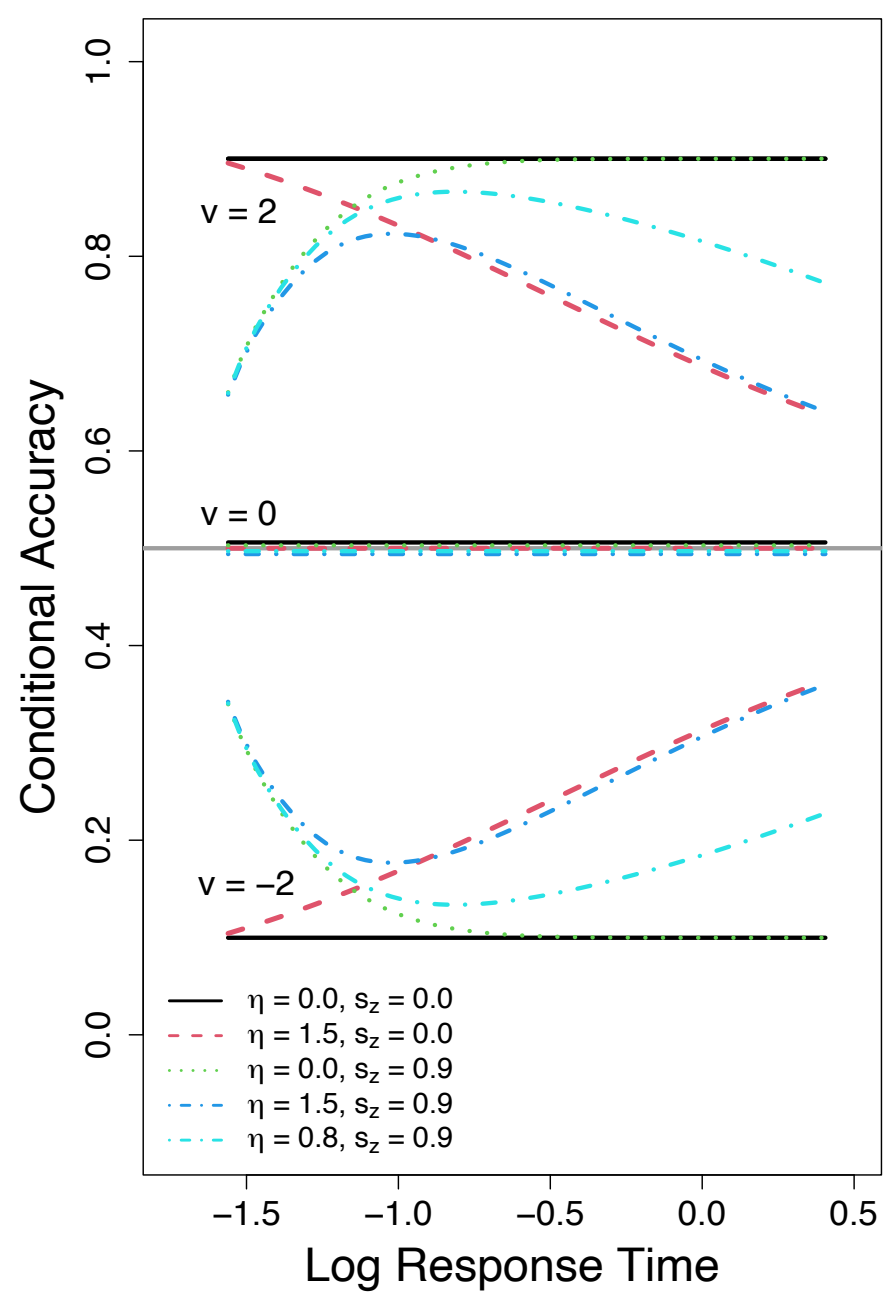

Figure 1: Conditional Accuracy Functions (CAFs) of the Ratcliff Diffusion Model. Figure adopted from Kang et al. (accepted for publication). The predicted CAFs are generated with varying values of across-trial variability in drift rates $(\eta)$ and in starting points $\left(s_{z}\right)$ shown at the bottom-left side of the figure. The other parameters used are boundary separation $\alpha=1.1$, starting point $z=\alpha / 2$, nondecision time $t_{0}=0.2$, and positive drift rate of $\nu=2$ for the top four curves (above the gray horizontal line indicating accuracy of 0.5), negative drift rate of $\nu=-2$ for the bottom four curves (below the gray curve), and zero drift rate $\nu=0$ for the four curves in the middle. Across-trial variability in nondecision time is set to 0 because it does not affect the shape of CAFs. The pure Wiener diffusion model (without variability parameters) predicts flat CAFs (black solid lines). With a positive drift rate, the model with nonzero $\eta$ predicts a decreasing CAF (red dashed line) while the model with nonzero $s_{z}$ predicts a CAF with an early steep increase (green dotted line). The model with nonzero variability parameters for both drift rate and starting point predicts curvilinear CAFs (dot-dashed lines). Among the two dot-dashed lines, the model with a larger value of $\eta$ predicts the one with a lower peak while the model with a smaller value predicts the one with a higher peak. CAFs predicted with a negative drift rate are the mirror images of those predicted with a positive drift rate, reflected over the gray horizontal line of accuracy $=0.5$. CAFs predicted with a zero drift rate are always flat. 
as the CAFs at the bottom side of Figure 1. The reason for the mirror image is that for negative drift rates, the diffusion process wanders off to the incorrect response boundary, which means that the information accumulation drifts away to an incorrect response, but with the same regularities as for a positive drift rate. When combined, the average across pairs of persons and items depends on whether the test is on average easy or difficult. It has been found earlier that the kind of dependency depends on the difficulty of the items. For a summary, see De Boeck and Jeon (2019). The opposite curvilinear shapes for easy and difficult tasks is in line with Chen, De Boeck, Grady, Yang, and Waldschmidt (2018b) using the same descriptive (not model-based) approach as in Chen et al. (2018a).

Summarizing, this leads us to a randomness approach to intelligence processes, with (1) an approximate approach for the drift rate component of the diffusion model, giving up on an isomorphic ambition, but with preserving the randomness character of information processing, and (2) an extra layer of randomness for two essential process parameters: drift rate and starting point. A randomness approach may seem to be an agnostic view on cognitive processes. Our view is different because randomness may be a realistic and important aspect of processes, and we do recognize some important aspects of processes, such as the use of prior knowledge, the natural oscillation of concentration, and the use of hypothesis-based inductive cognitive processes. Additionally, the second type of randomness has the potential to explain the repeatedly found residual dependencies between response accuracy and RT after controlling for respondent and item differences.

\subsection{Aim of the Study}

The primary purpose of our study is to throw further light on intelligence processes from a randomness perspective. To this end, we use the diffusion IRT models extended with randomness parameters because of their potential to understand the residual dependencies between response accuracy and RT discussed earlier. Kang et al. (accepted for publication) illustrated how to use these models to find model-based evidence for 
conditional dependency with exemplary item sets to measure 1) mental rotation ability and 2) extraversion (a personality trait). In the current study, we apply this modeling approach to two types of inductive intelligence tasks: verbal analogies and Raven type matrices; the same type of data have been used for studies of fast and slow intelligence (DiTrapani, Jeon, De Boeck, \& Partchev, 2016, Partchev \& De Boeck, 2012). It was found in a simulation study by Kang et al. (accepted for publication) that the diffusion IRT models with random variability parameters as used here could recover the model parameters well, using the same estimation method as for the present study. Therefore, we believe the proposed approach can be relied on to capture the hypothesized intricacies implied in the variability of the process components. The secondary purpose of our study is to investigate the relationship between speed and accuracy in terms of their overall individual differences. To be clear, the residual dependencies are dependencies after the overall item differences and individual differences are controlled for. The correlations between individual differences in speed and accuracy seem to depend on the type of task (Shaw, Elizondo, \& Wadlington, 2020; van der Linden, 2009). Apart from personality effects (Shaw et al. 2020), a possible explanation of the diversity of correlations across persons can be found in the SAT. Depending on the kind of tasks, respondents may choose different strategies to optimize their performance. For example, for some tasks it may pay off to spend time on the tasks to improve accuracy on the condition the respondent is able to improve accuracy, which may create a positive correlation between speed and accuracy. For other tasks it may not pay off because time does not help much, so that no correlation or a negative correlation can be expected between speed and accuracy. This could be the case for pure knowledge tests as one either has the knowledge or not, so that fast responses are more accurate and spending more time without having the knowledge, does not help.

The diffusion model (also its IRT variant) is a model with an SAT parameter; the larger the boundary separation is, the more emphasis is put on accuracy. As explained, how 
much emphasis is put on accuracy may depend on a combination of strategic considerations and one's capacity to make use of time on the task being solved. This would be shown as a rather high correlation between the boundary separation (a focus on accuracy) and the drift rate (capacity) across persons. Whereas one can interpret the SAT itself as a strategic choice (emphasizing speed or accuracy), one can see there also is room for a meta-strategy depending on whether an accuracy-based choice is profitable. To make an investment in accuracy profitable, the task needs to be one for which spending time is beneficial and the respondent needs to have the capacity to benefit from time investment. The correlation between boundary separation and drift rate can inform us on such meta-strategies and thus on what the basis is of SAT choices. It is also possible that the strategies and meta-strategies we refer to, are in fact learned habits or response styles one is not aware of. 


\section{Method}

\subsection{Model}

As mentioned in the introduction, we will employ a diffusion IRT model to study conditional dependency between response accuracy and RT of intelligence tests. Figure 2 illustrates the main structure of the model. For the response of person $p$ to item $i$, the model assumes that the person accumulates information over time at a mean rate $\nu_{p i}$. The person gives a response when the accumulated information exceeds one of the two decision boundaries, $\alpha_{p i}>0$ and 0 (upper and lower boundaries in Figure 2). For an intelligence test, the two boundaries represent the correct responses and the incorrect responses, respectively. The model predicts a response and its RT when the evidence accumulation terminates at one of the boundaries. The information is noisy and can tentatively evolve in favor of a response that is different from the response option the person eventually chooses and the chosen response can be different from the sign of the drift rate. Accordingly, the evidence accumulation is represented by noisy and wiggly trajectories as shown in Figure 2. This noise makes the model a probabilistic model, producing different responses and RTs even when the same set of parameters are used.

Cases 1-3 in Figure 2 show three possible random walk trajectories and eventual observed responses given the same boundary separation and drift rate. To illustrate these cases, suppose that the presented item is easy for the person and the person would likely choose the correct response option. In terms of model parameters, this means that the drift rate is positive and high. With this drift rate, the evidence accumulation process of the diffusion IRT model is likely to terminate at the upper boundary. Even in this case, the process can take shorter or longer to terminate, as shown by Cases 1 and 2. Also, even when the drift rate is positive, the process can terminate at the wrong decision boundary due to the random noise, as shown by Case 3. The model predicts an RT as a sum of decision time and nondecision time. The decision time is the time that the decision pro- 


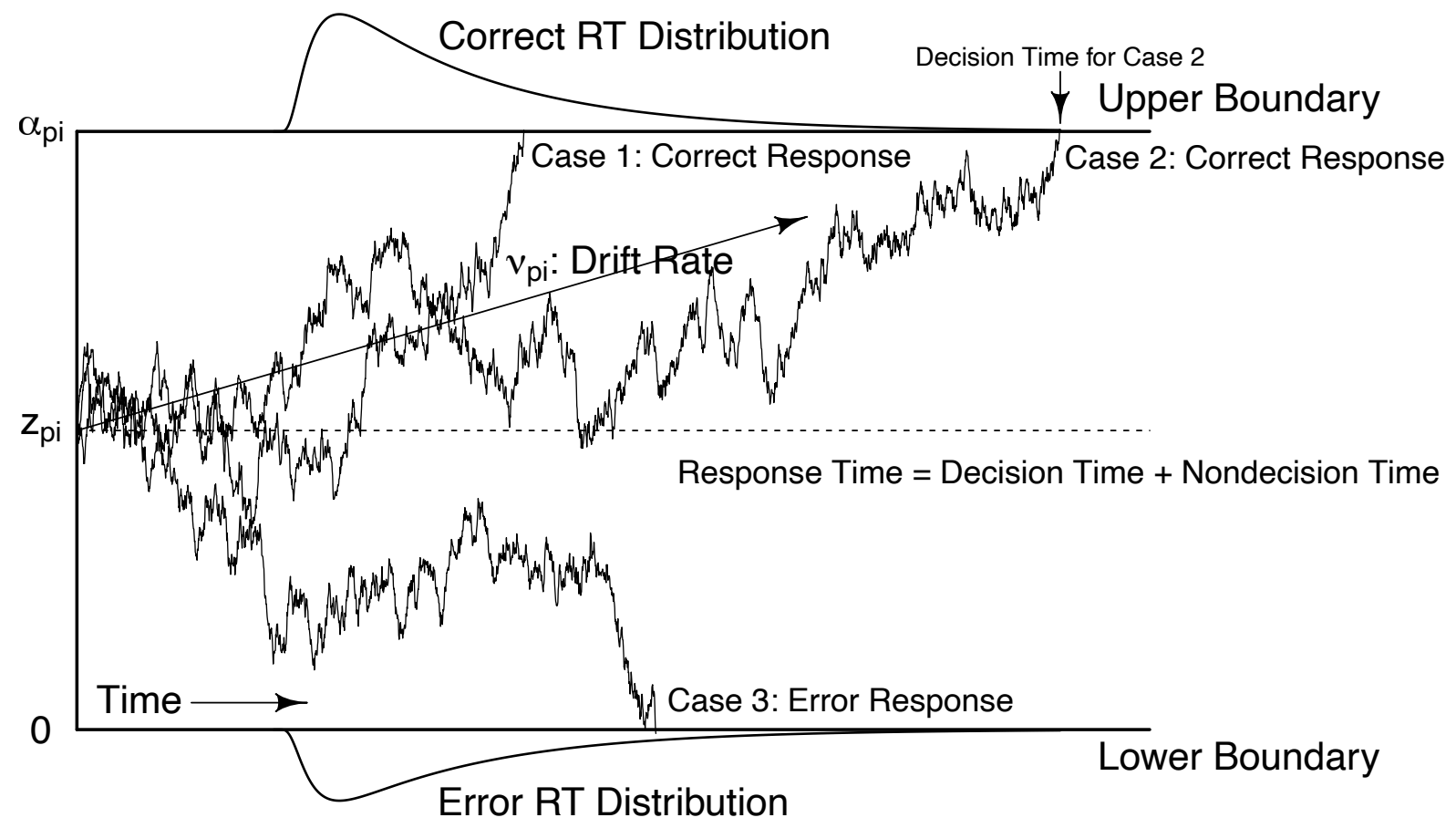

Figure 2: Diffusion IRT Model.

cess (evidence accumulation) takes to reach either boundary. For example, the decision time for Case 2 is shown at the top-right side of Figure 2. The nondecision time is the time taken for all cognitive processes not involved in the information accumulation process, which includes stimulus encoding, movement to make a response, etc., but not the cognitive work executed to find a response.

The model parameters represent different aspects of the processes involved. The mean rate of evidence accumulation $\nu_{p i}$ is called a drift rate and this represents the efficiency (quality) of information processing. For cognitive tests, this has been interpreted as a cognitive capacity (Bolsinova, Tijmstra, Molenaar, \& De Boeck, 2017; De Boeck et al., 2017). The distance between the two boundaries $\left(\alpha_{p i}\right)$ is called a boundary separation and this represents the amount (quantity) of information required to make a response. This also represents respondent $p^{\prime}$ s emphasis on accuracy when solving item $i$. The nondecision time represents all the time for processes unrelated to the information processing that leads to a response. There is another parameter, called the starting point $z_{p i}$, that indi- 
cates where the diffusion process starts between the two boundaries $\left(0<z_{p i}<\alpha_{p i}\right)$. It is the initial amount of information and thus it represents the initial bias of the response processes. However, it is commonly fixed to halfway the two boundaries of correct and incorrect responses in the diffusion IRT model approach (i.e., $z_{p i}=\alpha_{p i} / 2$ ) for the mathematical equivalence between the response proportion of the unbiased Wiener diffusion process and the two-parameter logistic IRT model (Tuerlinckx \& De Boeck, 2005).

To fit cognitive test data, the model should be able to decompose person and item effects, which turns the diffusion model into a diffusion IRT model. To this end, the following decompositions of the boundary separation (Molenaar, Tuerlinckx, \& van der Maas, 2015b; Tuerlinckx et al., 2016; van der Maas et al., 2011) and the drift rate (Molenaar et al. 2015b; Tuerlinckx \& De Boeck, 2005; Tuerlinckx et al., 2016) for person $p$ and item $i$ were implemented:

$$
\nu_{p i}=\theta_{p}-b_{i}, \alpha_{p i}=\gamma_{p} / a_{i}
$$

Here $\gamma_{p}$ and $\theta_{p}$ are person-wise emphasis on accuracy (a person parameter with an effect on the decision criterion) and person-wise capacity (a person parameter with an effect on the drift rate), respectively, and $a_{i}$ and $b_{i}$ are item time-pressure and item difficulty parameters, respectively. The first decomposition shows that the person-wise cognitive capacity relative to the item difficulty determines the drift rate per pair of a person and an item. The second decomposition shows that the person-wise emphasis on accuracy determines the actual decision boundary but reduced by the item-wise time-pressure. Also, in this paper, we assume person-wise nondecision time $\tau_{p}$ implying that different persons spend different amounts of time on nondecision processes. $1^{1}$

The drift rate decomposition in Equation 1 was originally proposed by Tuerlinckx and \begin{tabular}{|l|}
\hline \hline${ }^{1}$ Without variability in nondecision time (e.g., across-trial variability in nondecision time assumed in the \\
\hline Ratcliff diffusion model; Ratcliff, $2002 ; \mid$ Ratcliff \& Tuerlinckx, $2002 ; \mid$ Ratcliff \& McKoon, 2008), the estimation \\
\hline of the nondecision time is mainly based on the minimum RT. It was furthermore found that accounting \\
\hline for person-wise nondecision time can also control for inter-item differences in nondecision time as their \\
\hline contributions are confounded. The choice of having person-wise nondecision time instead of item-wise \\
\hline nondecision time is also motivated by a better decomposition of RTs into decision and nondecision times \\
\hline because typically the number of respondents is larger than the number of items in psychometric tests. \\
\hline
\end{tabular} 
De Boeck (2005), and is different from the one used later by van der Maas et al. (2011). They argued that abilities should be positive and so defined the drift rate as a quotient of person-wise capacity and item difficulty parameters with the requirement that these two parameters must be positive $\left(\nu_{p i}=\theta_{p} / b_{i}\right.$ with $\theta_{p}>0$ and $\left.b_{i}>0\right)$. In the present study, we use the earlier decomposition by Tuerlinckx and De Boeck (2005) instead of the positive ability assumption for the following reasons (Kang et al., accepted for publication). First, it remains to discuss whether the positive ability assumption is conceptually applicable to the drift rate. Although ability and drift rate have a positive association, drift rate is conceptually not equivalent to latent ability (Bolsinova, Tijmstra, Molenaar, \& De Boeck. 2017; De Boeck et al., 2017) and a negative drift rate can be interpreted as the tendency to move toward any of the wrong response options when an item is rather difficult for a respondent. Second, in common measurement models such as factor analysis models and IRT models, abilities are represented as latent variables with positive and negative values. Third, the parameterization by van der Maas et al. (2011) predicts that the lowest response accuracy should be 0.5 (unless further modifications are made), which is not suitable for difficult items with low accuracy. Accounting for such difficult items is particularly important for our modeling approach because they could have different patterns of residual dependencies as shown in Chen et al. (2018b) and also predicted by the extended diffusion models (Figure 1). Therefore, we work with drift rates that take values on the real line (and thus including negative values), as a majority of traditional psychometric models do for latent abilities.

The diffusion IRT model explained above assumes conditional independency: given the person and item parameters, response accuracy and RT are independent. It implies that the CAF is flat. However, it has been found that this assumption is violated for cognitive test data (Bolsinova, De Boeck, \& Tijmstra, 2017, Bolsinova \& Maris, 2016; Bolsinova \& Molenaar, 2018; Bolsinova, Tijmstra, \& Molenaar, 2017; Chen et al., 2018a; De Boeck et al., 2017: Goldhammer et al., 2014, 2015; Meng et al., 2015; Partchev \& De Boeck, 2012; van 
Rijn \& Ali, 2017; van der Linden \& Glas, 2010; Wang \& Xu, 2015) and the same authors also have presented models for the conditional dependency between response accuracy and RT.

Here we go one step further, from description to explanation. Instead of modeling the conditional dependency in a descriptive way, we will make use of several diffusion IRT models extended with random variations of process parameters. With these extensions, we will be able to explain the conditional dependency as the result of the random variations (as explained with Figure 1). Tuerlinckx and De Boeck (2005) and Kang et al. (accepted for publication) extended the model by introducing random variability in drift rate $(\eta)$ and in starting point $\left(s_{z r}\right)$, respectively. As shown in Equation 1, the drift rate $\nu_{p i}$ is determined as $\theta_{p}-b_{i}$ in the earlier diffusion IRT model; the extension with a random component implies that $\theta_{p}-b_{i}$ functions as the expected value and that a normally distributed random coefficient is added with a mean of zero and a standard deviation (SD) $\eta$. For the starting point, the extension implies that $z_{p i}$ (set equal to $\alpha_{p i} / 2$ ) functions as the expected value of the starting point and that a random coefficient is added which is assumed to follow a uniform distribution with range $\alpha_{p i} \cdot s_{z r}$. Parameter $s_{z r}$ indicates the size of the variability and is bounded by 0 and 1 , so that the starting point cannot move beyond the boundaries.

For similar previous extensions with random variability (Kang et al., accepted for publication; Tuerlinckx \& De Boeck, 2005), only a single parameter for each of the two random variability components was employed $\left(\eta\right.$ and $\left.s_{z r}\right)$, meaning that the overall amount of variation and overall degree of conditional dependency are equivalent for all persons and items. This might be too restrictive, as it has been shown that the conditional dependency can be item-specific (Bolsinova, De Boeck, \& Tijmstra, 2017; De Boeck et al. 2017). Given a sufficient number of persons, it may be possible to estimate item-wise variability parameters, $\eta_{i}$ and $s_{z r, i}(i=1, \ldots, I)$, which is an even further and new extension of the diffusion IRT model. Although variability parameters are relatively more 
difficult to estimate with precision (Ratcliff \& Tuerlinckx, 2002; Ratcliff \& McKoon, 2008), we will also attempt to estimate item-wise variability parameters because our samples are large. Although Kang et al. (accepted for publication) have provided a simulation study to show parameter recovery of the diffusion IRT models with random variability components, our extension with item-wise variability parameters is new and needs to be further examined. In Appendix C, we provide Kang et al.'s simulation study to show that item-wise variability parameters can be reasonably recovered. If the number of items would be much larger than for our data, person-wise variability parameters could also be considered. However, it is uncommon to have large item sets, and for our study, the numbers of items per persons are only 24 and 11, so that we will not estimate person-wise variability parameters.

\subsection{Data}

For the primary and the secondary research purpose, we rely on the same data: two datasets, one with verbal analogy item responses and another with matrix reasoning item responses. Each of the data sets stems from the same large pool of data (collected for a large-scale project in the German military) also used in DiTrapani et al. (2016) and Partchev and De Boeck (2012). For matrix reasoning, we have used the same subset of data as in the previous studies and for verbal analogies, our data set turned out to be a somewhat larger subset. We have no explanation for why the earlier used subset for verbal analogies was somewhat smaller. A more extensive description of the data than in the following, is provided in the two previous articles. For each dataset, we have two data matrices, one for responses and the other for RTs, with rows of the matrices corresponding to persons and columns corresponding to items. All items are multiple-choice items, with five response options for the verbal analogy items and eight response options for the matrix items. The responses are coded 1 or 0 , representing correct or incorrect responses, respectively. RTs are recorded in seconds. The items for the two tests we investigate here 
were developed by Hornke and colleagues. For more detailed descriptions on the item construction, we refer readers to Hornke (1999, 2001), Hornke and Habon (1986), and Hornke and Rettig (1993).

For the current study, the verbal analogies data concern responses from $P=904$ persons and $I=24$ items, with some missing data. The overall mean accuracy is 0.729 , ranging across items from 0.220 to 0.963 with a median of 0.800 . The $10 \%, 30 \%, 50 \%, 70 \%$, and $90 \%$ RT quantiles based on all responses are 7, 11, 15, 20, and 35 seconds, respectively. Some responses have extremely long RTs, which potentially are outliers. Responses with RTs longer than 90 seconds are excluded in the analysis, which represents $0.6 \%$ of the total number of responses. The matrix reasoning data concern $P=504$ persons and $I=35$ items. The data were collected with a block design such that not each person was presented with all items. On average, each person responded to $11.1(\mathrm{SD}=1.0)$ items and responses from $159.2(\mathrm{SD}=46.2)$ persons are available for each item. The overall mean accuracy is 0.452 , ranging across items from 0.025 to 0.873 with a median of 0.462 . The five RT quantiles are 22, 38, 55, 79, and 131 seconds, respectively. Although there was the item-wise time limit of 180 seconds (Hornke \& Wilding, 1997), about $4 \%$ of the total cases have RTs longer than the time limit. These cases are not included in the main analysis. One respondent answered all items within 1-5 seconds, which is potentially a fast random guessing respondent. This respondent is also excluded in the main analysis, leaving 503 persons.

\subsection{Data Analysis Approach}

In the first step of the analysis, we compare different diffusion IRT models, with and without variability components. The model without any variability components functions as a reference, which is a model assuming conditional independency. If models with variability components fit the data better than the reference model, this provides modelbased evidence for the presence of conditional dependency. To study potential sources 
of conditional dependency, different variability models are defined and compared. The models are denoted by their variability component. The three models with variability are models $(\eta),\left(s_{z r}\right)$, and $\left(\eta, s_{z r}\right)$ (see Table 1). In the second step, these three models are extended with item-specific variability components of the drift rate and starting point: models $\left(\eta_{i}\right),\left(s_{z r, i}\right)$, and $\left(\eta_{i}, s_{z r, i}\right)$. Similarly to the models from the first step, these models allow for random deviations in drift rates and/or in starting points across pairs of persons and items, but now with an item-specific size of the variability. If these extensions improve the model fit to a large degree, the improvement provides evidence for item-specific conditional dependency as found in De Boeck et al. (2017).

Independent of the variability components, all these models also provide us with estimates of the person parameters so that their correlations can be studied. For the person parameters, we use a hierarchical (prior) distribution so that person-wise cognitive capacity $\left(\theta_{p}\right)$ and log-transformed emphasis on accuracy $\left(\log \left(\gamma_{p}\right)\right)$ are normally distributed with mean $(0,0)$, variances $\left(\sigma_{\theta}^{2}, \sigma_{\gamma}^{2}\right)$, and covariance $\sigma_{\theta \gamma}$. For the other parameters, priors are given based on the findings in earlier studies with the diffusion IRT models (e.g., Kang et al. accepted for publication: Molenaar et al., 2015b). With these prior specifications, we implement a Bayesian approach to estimate models as described in Appendix A.

For all model comparisons, we base our conclusion on the modified Akaike Information Criterion (mAIC) and the modified Bayesian Information Criterion (mBIC; Bolsinova, Tijmstra, \& Molenaar, 2017; Bolsinova \& Molenaar, 2018, 2019). These are versions of the conventional AIC (Akaike, 1974) and BIC (Schwarz, 1978), proposed for models estimated with a Bayesian method and can be calculated by adding a penalty term to -2 log-likelihood (-2LL) evaluated at the posterior means of the model parameters. These modified criteria were proposed as an alternative to the Deviation Information Criterion (DIC; Spiegelhalter, Best, Carlin, \& Van Der Linde, 2002) for some complex models such as highly nonlinear models and mixture models (e.g., Molenaar \& De Boeck, 2018) because the DIC worked suboptimally for these models (e.g., preferring other models to the data- 
generating model). The modified criteria have been utilized for joint models of responses and RTs in earlier studies (Bolsinova, Tijmstra, \& Molenaar, 2017; Bolsinova \& Molenaar, 2018, 2019). Although the mAIC and mBIC can help us conduct a model comparison, the best fitting model is not necessarily a well-fitting model. To evaluate absolute model fit, we examine whether the model prediction of accuracy and RT distributions matches the data. If a model fails to predict response accuracy and RT distributions, it cannot be used to answer our research questions related to the primary and secondary purposes of the analysis.

Remember that for the conditional dependencies, the CAFs predicted by the model are of primary interest. The CAF is defined as the conditional probability that a response is correct given the RT and person and item parameters (Kang et al., accepted for publication: Luce, 1986). The CAF shows accuracy as a function of RT given the latent variables and item parameters, and thus, displays the trend of conditional dependency between response accuracy and RT. When response accuracy and RT are conditionally independent, the CAF is flat. The diffusion IRT model with random variability accounts for conditional dependency, producing increasing, decreasing, or curvilinear CAFs (Kang et al., accepted for publication). The model predicts a CAF underlying person $p$ 's response to item $i$, producing $P \times I$ CAFs for data with $P$ persons and $I$ items. With these predicted CAFs, we can study general patterns of conditional dependency and heterogeneity across persons and items. Details of the model fitting method and the prediction generation method can be found in Appendices A and B. 


\section{Results}

\subsection{Model Comparison}

Table 1 shows the relative model fits evaluated based on deviance measures such as -2 log-likelihood (-2LL), mAIC, and mBIC. For the verbal analogies data, the full model with item-wise variability in drift rate and in starting point $\left(\eta_{i}, s_{z r, i}\right)$ performs the best. Compared to the reference model (no variability model), the models with variability parameters have improved values of the model fit indices, indicating that response and RT are conditionally dependent. Variability in drift rate improved the model fit to a large degree whether it consists of a general variability parameter for all person and item pairs (i.e., $\eta$ ) or item-wise variability parameters $\left(\eta_{i}\right)$. A general variability in starting point $\left(s_{z r}\right)$ did not improve the model fit but allowing item-specific variability $\left(s_{z r, i}\right)$ produced much better model fits, particularly when implemented with item-wise variability in drift rate (i.e., the full model with $\eta_{i}$ and $s_{z r, i}$ ).

For the matrix reasoning data, the full model has a convergence issue with itemspecific variability in drift rate. This might be attributed to the fewer number of observations $(P=503$ and $I=35$, but on average 11.1 item responses per person and 159.2 respondents per item) compared to the verbal analogies data $(P=904$ and $I=24$ with only a few missing values). In particular, there were some items with a few respondents (minimum of 85 ) due to the randomized design used in the data collection, which made it difficult to obtain a convergent result for the full model (see Appendix $C$ for a related discussion regarding a simulation study). Among the two other models with item-specific variability parameters, the model with item-wise variability in starting point $\left(s_{z r, i}\right)$ shows the best fit. The models without item-specific variability or without any extra variability (i.e., the reference model) have a clearly lower goodness of fit. As will be shown, the absence of a random component in the drift rate in the $\left(s_{z r, i}\right)$ model has consequences for the shape of the conditional dependency. 


\begin{tabular}{cccccccc}
\hline Model & DIRT & $(\eta)$ & $\left(s_{z r}\right)$ & $\left(\eta, s_{z r}\right)$ & $\left(\eta_{i}\right)$ & $\left(s_{z r, i}\right)$ & $\left(\eta_{i}, s_{z r, i}\right)$ \\
\hline \multicolumn{2}{l}{ Verbal Analogies } & & & & & & \\
-2LL & 158467.1 & 158143.7 & 158462.1 & 158187.7 & 157949.4 & 158260.7 & $\mathbf{1 5 7 4 4 5 . 8}$ \\
mAIC & 163993.1 & 163671.7 & 163990.1 & 163717.7 & 163523.4 & 163834.7 & $\mathbf{1 6 3 0 6 7 . 8}$ \\
mBIC & 177274.4 & 176957.8 & 177276.2 & 177008.5 & 176920.0 & 177231.3 & $\mathbf{1 7 6 5 7 9 . 8}$ \\
& & & & & & & \\
\multicolumn{2}{l}{ Matrix Reasoning } & & & & & & \\
-2LL & 55025.0 & 55036.3 & 55025.2 & 55063.2 & 54904.2 & $\mathbf{5 4 7 6 8 . 7}$ & - \\
mAIC & 58189.0 & 58202.3 & 58191.2 & 58231.3 & 58138.2 & $\mathbf{5 8 0 0 2 . 7}$ & - \\
mBIC & 63045.7 & 63062.0 & 63050.9 & 63094.2 & 63102.4 & $\mathbf{6 2 9 6 6 . 8}$ & - \\
\hline
\end{tabular}

Table 1: Relative Fit Indices. The values of 1) overall fits of the models evaluated with -2 log-likelihood (-2LL), the modified Akaike information criterion (mAIC), and the modified Bayesian information criterion (mBIC) are shown. The first column shows the model fits of the DIRT model. The other columns show the model fits of the DIRT models with different random variability parameters as specified in the leading row. DIRT: Diffusion IRT model.

For both, the verbal analogies data and matrix reasoning data, there were some other models we examined but omitted in Table 1, such as the model with a single variability in drift rate and item-wise variability in starting point $\left(\eta, s_{z r, i}\right)$ and the model with itemwise variability in drift rate and a single variability in starting point $\left(\eta_{i}, s_{z r}\right)$. Due to the inter-item differences in conditional dependency discussed above, these combinations of the variability parameters did not improve or even worsened the model fits, so that we did not investigate these models further.

The next question is whether the best-fitting model does sufficiently cover the observed data (i.e., absolute model fit). This is important because, without a good absolute fit, we cannot rely on the model predicted CAFs. As shown in Appendix B, the absolute goodness of fit is satisfactory, except for some uncertainties in the error RT distributions of the verbal analogies data. This was because some items were rather easy and there were relatively a few error observation for these items. Therefore, data error RT quantiles cannot be precisely obtained and the estimation program weigh more on correct responses and their RTs, potentially producing some misfit in the error RT distribution. The satisfactory absolute goodness of fit for the matrix data implies that the $\left(s_{z r, i}\right)$ model can provide 
us a satisfactory view of the underlying processes.

\subsection{Conditional Accuracy Functions}

The relative and absolute goodness of fit of the models with random variation of drift rate and starting point have provided evidence for the presence of conditional dependency between response accuracy and RTs for both of our two data sets. Given these results, we can further investigate the shape of the conditional dependency by visualizing the dependency with model-predicted CAFs. Figure 3 shows the CAFs for the verbal analogies data (left) and the matrix reasoning data (right) predicted by their best-fitting models. Each panel shows $P \times I$ CAFs as gray curves, each of which corresponds to the combination of a person and an item.

To interpret the curves, it helps to explain the consequences of the random variation of the starting point and the drift rate. If the starting point has a random variation component, the starting point may be located in the opposite direction of the drift rate. In such a case, it may take time for a positive drift rate process to catch up and reach the correct response boundary. It is also possible that by accident, due to its stochastic nature, the drift process ends up at the incorrect response boundary, which lowers the probability of a fast response to be correct. Such an outcome is less likely for a longer positive drift process. This explains the upward CAFs. For a negative drift rate, the mirror image of the upward CAF can be expected, because the process is geared to the opposite direction. This explanation corresponds to the CAFs for the matrix reasoning (as shown in the right panel of Figure 3) as the best-fitting model with good absolute fit to the matrix reasoning data was the model with item-wise variability in starting point but no variability in drift rate.

The situation is more complex when there also is a random variation in drift rate. If the process is geared to the correct response boundary (i.e., with a positive drift rate), the variation will create a negative residual correlation between response accuracy and RT, 
because a positive drift rate reduces $\mathrm{RT}$ and increases the accuracy rate. If the process is geared to the incorrect response boundary, the variation will create a positive residual correlation (i.e., the mirror image). This kind of dependency is in line with findings by De Boeck et al. (2017) and Bolsinova, De Boeck, and Tijmstra (2017) that the dependency is related to the difficulty of the items and is less negative and even positive for more difficult items. The combination of the two types of variation, i.e., of the starting point and of the drift rate leads to curvilinear shapes. This explanation corresponds to the CAFs for the verbal analogies (as shown in the left panel of Figure 3) as the best-fitting model for the verbal analogies data was a model with item-wise random variability in starting point and in drift rate. Not surprisingly, Chen et al. (2018b) has found a similar curvilinearity and mirror images when in a follow-up study the results as presented in Chen et al. (2018a) are split up into results for easy and difficult items. It is important that the latter results are obtained with different and partly knowledge-based test data and that they are not based on any model estimation. Instead, they are purely descriptive results obtained with a data exploration method after controlling for main effects of persons and items. It means that our curvilinear results cannot be explained away as induced by the extended IRT drift diffusion model and that instead, the model captures an empirical reality.

As can be seen in the left panel of Figure 3, for the verbal analogies data, the predicted CAFs for relatively easier items (with accuracy higher than 0.5 ) show a clear curvilinear trend as accuracy goes up in the fast RT range and then decreases over time in the slow RT range. This is consistent with the above explanation and with the previous findings in Bolsinova and Molenaar (2018), Chen et al. (2018a), and Kang et al. (accepted for publication). Also, as the overall accuracy was moderately high (accuracy of 0.731 ) and many persons and items had a high accuracy level, most CAFs are located in the upper half of the panel and sometimes near the top, producing a ceiling effect as one can notice in the darker area of the panel. In general, the curvilinear shape implies that responses with RTs faster than the expected values are relatively slightly less accurate and that responses with 
RTs slower than the expected values are also relatively less accurate. Kang et al. (accepted for publication) showed that, for relatively easy items that are associated with positive drift rates, the model predicts an early increasing pattern with the starting point variability and thus its primary source is the initial bias in the response processes. Since verbal analogies require vocabulary knowledge as well as inductive reasoning, the starting point can be interpreted as the initial hypothesis of the inductive reasoning process. The model also predicts a later decreasing pattern for relatively easy items with the variability in drift rate and thus the downward part of the curve can be explained by the variation in drift rate, which either stems from the specificity of knowledge or a natural variation of cognitive capacity resources. For relatively difficult items (with accuracy lower than 0.5), the predicted CAFs are generally the mirror images of the CAFs for the relatively easy items. As shown in Kang et al. (accepted for publication), the model predicts this pattern when drift rate is negative and variability in drift rate is nonzero. Thus, this can be attributed to the variation in drift rate. Note that even when words as such are known, it does not imply that respondents are aware in an explicit way of all features necessary to solve analogy problems when they encounter a word on the test. These can be features such as polysemic and semantic features. For example, the word "bachelor" has different meanings and the meanings come with defining features (e.g., male and unmarried) that may help solve analogy problems. These person-by-word differences in pre-knowledge for word features and different meanings can produce the variation in drift rate.

The predicted CAFs for the matrix reasoning data show completely different patterns. Over the RT range until the time limit, the CAFs are increasing for relatively easy items and are decreasing for relatively difficult items. The item-wise starting point variability in the best-fitting model is the dominant source of conditional dependency for the matrix data, and thus, these patterns should be attributed to the random variation of the starting point. The tasks require the respondents to go through a process of sequential hypothesis testing with a random start for the initial hypotheses. The good absolute fit of the best- 

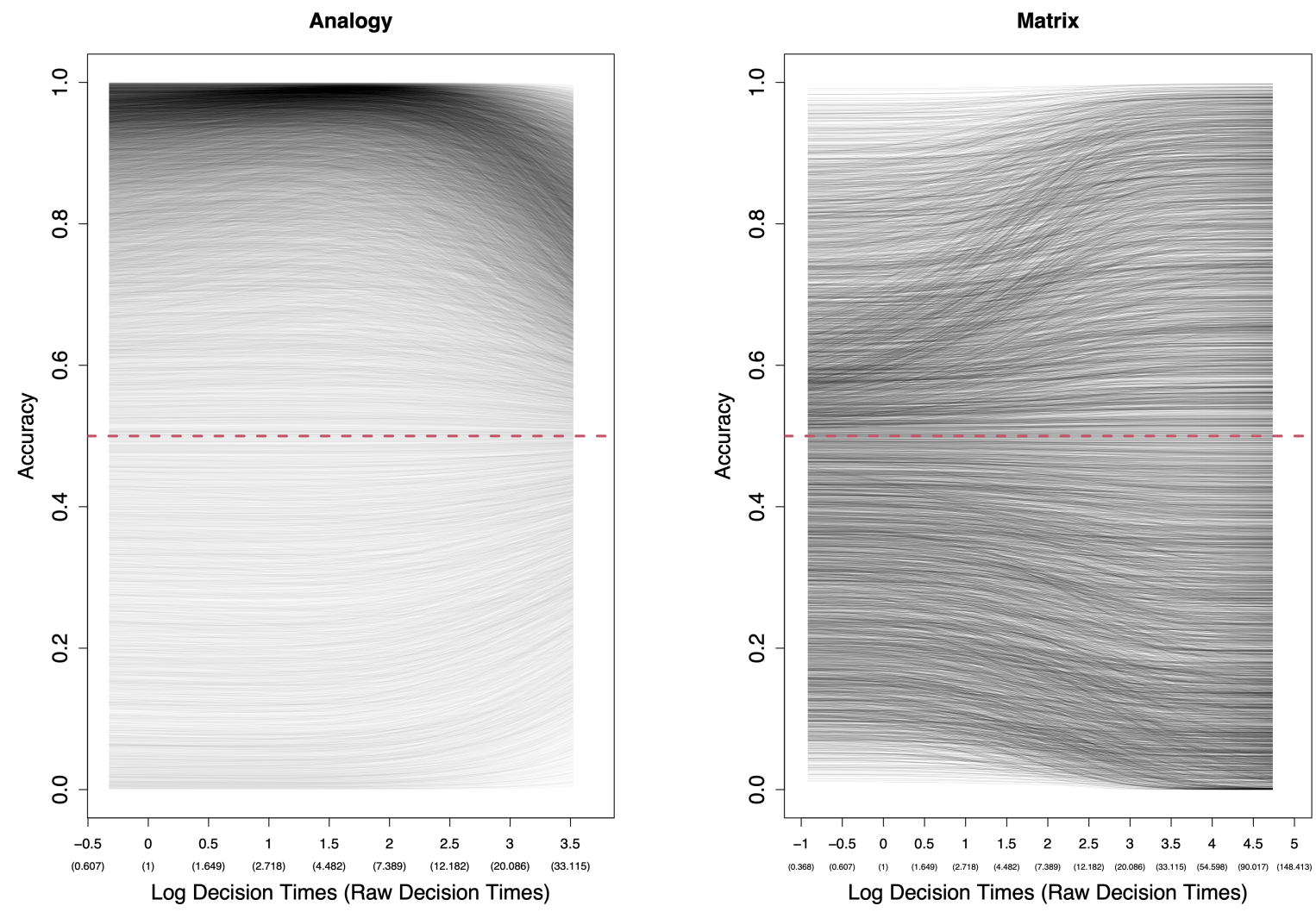

Figure 3: The Predicted Conditional Accuracy Functions (CAFs) for the Verbal Analogies Data (Left) and the Matrix Reasoning Data (Right): In each panel, each black curve shows a CAF corresponding to one of the $P \times I$ person-item combinations (i.e., responses).

fitting model assures that this interpretation based on the cognitive components of the model provides a trustworthy view on the CAFs.

To provide a clearer look at the CAFs, particularly regarding across-person or acrossitem heterogeneity in conditional dependency, some persons and items are selected as examples and their CAFs are plotted in Figure 4. The panels on the left and on the right show the examples for the verbal analogies data and the matrix reasoning data, respectively. In the top two panels, three persons with different overall accuracy (across items) are selected and their CAFs for all items are plotted. In the bottom panels, three items with different overall accuracy (across persons) are selected and their CAFs for all persons are plotted. This figure shows that even the same person (same item) can show curvilinear, increasing, decreasing, or flat CAFs for different items (different persons). For example, 
Person 880 in the verbal analogies data (the blue curves in the top-left panel) has curvilinear CAFs for relatively easy items, flat CAFs for items with the accuracy close to 0.5, and generally increasing CAFs for difficult items. Therefore, behavioral patterns of the conditional dependency can differ by how difficult an item is for a person (i.e., person and item effects). Person-wise CAFs in the matrix reasoning results (the top-right panel) show that persons with high accuracy (the black curves) can profit from taking more time to solve a problem but this may not be the case (some of the red curves) or even the reverse can be the case (some of the blue curves) for persons with low accuracy. Item-wise CAFs generally agree with person-wise CAFs. The CAFs in the bottom-right panel show that accuracy tends to increase over time for an easy item while it decreases for a difficult item. This might be because the test items are generally rather difficult (overall accuracy of 0.452 ) and so the item-wise time limit of 180 seconds was perhaps too short for some highly difficult items. Although the respondents almost always has responded within the time limit (except for only $4 \%$ of the responses), they may have spent less effort in the later stages of working on a difficult item. For relatively easy items, persons can reach the correct response option by processing more time. 

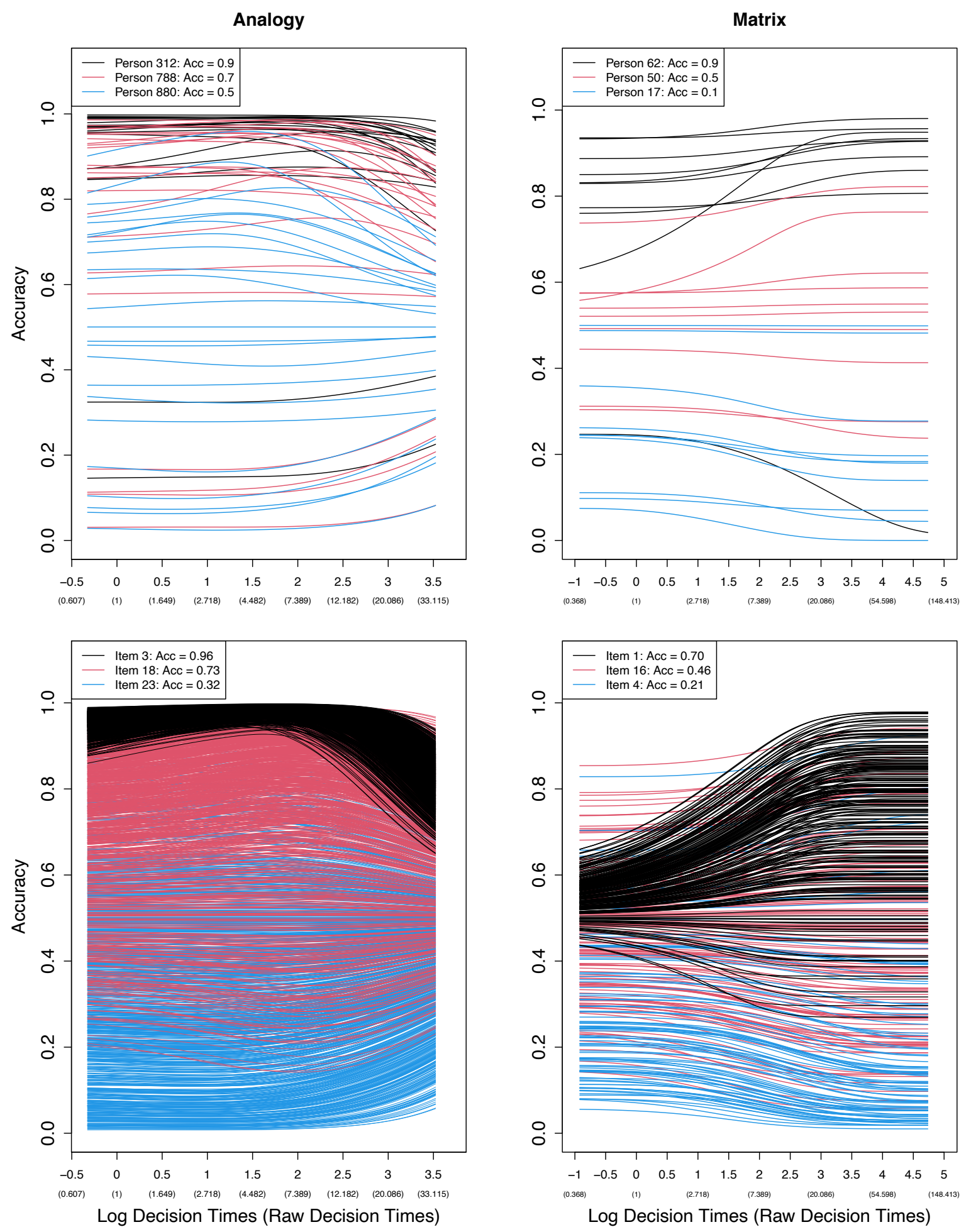

Figure 4: Some Selected Examples of the Predicted Conditional Accuracy Functions (CAFs) for the Verbal Analogies Data (Left) and the Matrix Reasoning Data (Right): The top (bottom) row shows the predicted CAFs of three selected persons (items), with different levels of accuracy as shown at the top-left side in each panel. 


\begin{tabular}{l|rcccc}
\hline & 1 & 2 & 3 & 4 & 5 \\
\hline 1. Accuracy & & 0.467 & 0.278 & 0.926 & 0.644 \\
2. Person Mean Log RT & -0.075 & & 0.015 & 0.627 & 0.818 \\
3. Person SD Log RT & 0.091 & 0.176 & & 0.360 & 0.412 \\
4. $\theta_{p}$ & 0.960 & -0.167 & 0.060 & & 0.777 \\
5. $\log \left(\gamma_{p}\right)$ & 0.245 & 0.814 & 0.466 & 0.164 & \\
\hline
\end{tabular}

Table 2: Correlations Across Persons. Values below the diagonal line are the Pearson correlations among the person-wise parameters and descriptive statistics for the verbal analogies data while values above the diagonal lines are those for the matrix data.

\subsection{Correlational Results}

The correlations among model parameters for persons are reported in Table 2 . In the table, values below the diagonal line are the Pearson correlations among the person-wise parameters and descriptive statistics (mean accuracy and mean and standard deviation of $\log$ RTs) for the verbal analogies data while values above the diagonal lines are those for the matrix data. For verbal analogies, the correlation between mean accuracy (proportion of correct responses) and mean log RT (mean of log-transformed RTs) is near zero $(-0.075)$, and each of these two statistics is highly correlated with a model parameter. Accuracy is highly correlated with drift rate $(0.960)$ and mean $\log \mathrm{RT}$ is highly correlated with boundary separation (0.814). Also, the partial correlation between accuracy and drift rate given boundary separation is 0.962 and the partial correlation between mean log RT and boundary separation given drift rate is 0.865 . Thus, the very high correlations between accuracy and drift rate and between boundary separation and mean log RT are independent of the other person-wise component. It means that, as far as individual differences are concerned, the SAT choice (i.e., boundary separation) is the driving force of RT and cognitive capacity (i.e., drift rate) is the driving force of accuracy. The correlation between boundary separation and accuracy and their partial correlation controlling for drift rate are low ( 0.245 and 0.318 , respectively). Together, these results mean that an accuracy-based measure primarily reflects capacity and is rather independent of individual differences in SAT. 
For matrix reasoning, the correlation between mean accuracy and RT is moderately positive (0.467). Although each of the two statistics is highly correlated with the same model parameter as for verbal analogies ( 0.926 for accuracy and drift rate, and 0.818 for mean log and boundary separation), there also are rather high cross-correlations (0.644 and 0.604), and the correlation between the two model parameters is also high (0.777). These correlations can be interpreted as an illustration of the meta-strategical principle. Matrix reasoning tasks have a rather long RT and may require sequential hypothesis testing, which would pay off more for respondents who are skillful at generating, testing, and keeping track of subsequent intermediate induction results, so that investing in time is extra beneficial for accuracy. The high correlation also implies that less capable persons may not be able to profit or even loose accuracy while taking more time. The upper-right panel of Figure 4 corresponds to this interpretation in that conditional accuracy increases over time only for a person with high accuracy while it decreases for a person with low accuracy. The consequence of the high positive correlation between the two model parameters, is that the correlation between accuracy and speed also is positive, in contrast with verbal analogies. With respect to possible distortions of measurement, the rather high correlation of accuracy with boundary separation (0.644) is less of a problem, knowing that the correlation of accuracy with drift rate is extremely high (0.926) and boundary separation may be induced by the person's drift rate as their correlation (0.777) implies. The partial correlation between accuracy and boundary separation, controlling for drift rate is low $(-0.318)$, so that also for matrices, an accuracy-based measure reflects cognitive capacity in the first place.

Together the results for verbal analogies and matrix reasoning show that the individual differences correlation between speed and accuracy can be task-dependent, as was already known from the literature (e.g., Shaw et al., 2020; van der Linden, 2009). It is possible that the SAT choice of respondents is sometimes driven by their capacity (matrix reasoning) and sometimes it is an independent choice (verbal analogies). The results sug- 
gest the possibility of a return-on-investment meta-strategy, which entails that investing in time and using a high response threshold depends on having the capacity for a return on investment in terms of accuracy. 


\section{Discussion}

In this article, we studied intelligence processes from a randomness perspective: intelligence processes have some irregular and fluctuating aspects that cannot be fully accounted for by systematic person and item effects. The randomness aspects were implied in two ways. First of all, the random walk nature of the information processing as illustrated in Figure 2 implies that solving processes are not straight paths from start to end. Second, there seems to be random variation across persons and items, which leads to conditional dependencies between responses and RTs (after controlling for person and item parameters). Random variability in the diffusion IRT models can explain the findings from earlier studies with cognitive test data (Bolsinova, De Boeck, \& Tijmstra, 2017, Bolsinova \& Maris, 2016; Bolsinova \& Molenaar, 2018; Bolsinova, Tijmstra, \& Molenaar, 2017; Chen et al. 2018a; De Boeck et al., 2017; Goldhammer et al., 2014, 2015; Meng et al., 2015; Partchev \& De Boeck, 2012; van Rijn \& Ali, 2017; van der Linden \& Glas, 2010; Wang \& Xu, 2015). The random nature of the processes, even for the high ability range, is somewhat counter-intuitive given the association of intelligence with reasoning. To shed light on these random features of intelligence processes, we made use of the diffusion IRT models extended with random variability parameters (Kang et al., accepted for publication). This approach is in line with the growing number of diffusion model approaches that aimed to study intelligence processes with cognitive/mathematical models that provide a theoretical and explanatory view of psychological processes underlying cognitive tests. (Frischkorn \& Schubert, 2018; Lerche et al., 2020; McKoon \& Ratcliff, 2012, Ratcliff et al., 2008, 2009, 2011; Schmiedek et al., 2007; Schulz-Zhecheva et al., 2016; Schmitz \& Wilhelm, 2016: Schubert \& Frischkorn, 2020; Schubert et al., 2015). An important difference between the previous studies and our current work is our focus on remaining dependencies and an approach based on random variability parameters, with the potential to investigate intelligence processes more in detail based on a more microscopic approach.

We analyzed verbal analogies data and matrix reasoning data with an extended dif- 
fusion IRT model and studied conditional dependency between responses and RTs of intelligence tests. Our full model includes variability in drift rate and in starting point which represent the variation in cognitive capacity and the variation in initial bias of the response processes, respectively. Drift rate variability is known to produce negative dependency for easy items and positive dependency for difficult items, capturing the different behavioral patterns of conditional dependency moderated by item difficulty. Starting point variability also produces increasing conditional accuracy as a function of RTs, but mostly in the faster than expected RT range. Therefore, the combination of both variability components accounts for the curvilinear conditional dependency that has previously been found in Bolsinova and Molenaar (2018) and Chen et al. (2018a). The variability parameters can also be allowed to vary by item to account for the item-specific nature of dependency (De Boeck et al., 2017). The fact that the diffusion IRT model with random variability can explain these phenomena, which are descriptive but unexplained findings from earlier studies, lends credibility to our model to investigate intelligence processes.

For the verbal analogies data, the best-fitting model was the full model with item-wise variability in drift rate and in starting point. The model showed a much goodness of fit compared to the model without any variability component. The dominant trend in the CAFs predicted by the combination of item-specific variability in drift rate and in starting point assumed in the best-fitting model was curvilinear with an early increase and later decrease of accuracy as a function of time. Because the accuracy rate of the test responses was rather high, the average shape of the CAFs was better represented by the upper half of the left panel in Figure 3. The shape is perfectly in line with the shapes found by Bolsinova and Molenaar (2018) and Chen et al. (2018a) who have worked with rather high success rates of responses. To interpret the result in terms of intelligence processes, it is important to notice that verbal analogies are inductive tasks and require vocabulary knowledge. Word knowledge comprises more than knowing and understanding the word. It also comprises more elaborate word features (e.g., polysemic and semantic fea- 
tures) that may help to solve analogy problems. Word knowledge is to some extent specific to the pair of a person and an item, in the sense that after controlling for global word knowledge as captured by the person's and item's overall drift rate contribution, there are remaining item-specific differences between individuals. This type of variation can explain the item-specific drift rate variation. Because verbal analogies also are inductive tasks, we may also expect sequential hypothesis testing process to find the rules underlying the analogies, and these hypothesis testing processes imply random variation in the starting point. Also, the initial bias of the response process can differ across persons and items depending on responses to earlier items, which would be another potential source of variability in starting point. The two random variation components are informative for the kind of processes involved in verbal analogy tests.

For the matrix reasoning data, it was not possible to obtain a convergent model fitting result for the model with two sets of item-specific variability parameters. The best-fitting model was the one with item-wise variability in starting point and no variability in drift rate. Because of a convergence issue, we do not have results from the full model with two item-wise random variability components (not just for starting point but for drift rate as well). However, the absolute fit of the model with just item-wise variability in the starting point seems satisfactory. A possible explanation is that matrix reasoning does not require acquired knowledge and is based on hypothesis testing with - to some extent - a random staring point.

For both types of tasks, our findings offer an explanation for the remaining dependencies between response accuracy and RT by assuming the random variation of IRT diffusion model parameters. The random variation is more than just a statistical extension of the model; it also makes sense from a cognitive processes point of view. Knowledge and information processing is to some extent specific across persons and items and attention and concentration may also fluctuate during the test. Both these sources can be captured through random variation component of drift rate. Also, realistically speaking, 
starting point and progress of processes is to some extent random, which are captured by the random variation component of the starting point and by the stochastic nature of the information accumulation process, respectively. Our results suggest that intelligence processes show some randomness and are not just rational, insightful, and smart reasoning (i.e., can deviate from what is systematically expected from person and item effects).

Regarding the secondary purpose of our study, the correlation between individual differences in response accuracy and RT seems to depend to a large extent on the nature of the task. The individual differences in boundary separation are highly and positively correlated with the individual differences in drift rate for matrix reasoning but not for verbal analogies. These findings suggests that a focus on accuracy may depend on one's capacity. The SAT of a respondent can be interpreted as a strategy and it may depend on one's capacity to find which strategy pays off more. This further implies that more capable persons can benefit more from processing more time while less capable persons may not or even the reverse can be the case. The predicted CAFs of some selected respondents in the matrix reasoning test (the upper-right panel of Figure 4 ) agree with this interpretation. Although our evidence is purely correlational, it suggests a dynamic, personal, and expected return-based reality underlying the SAT for intelligence processes. It can be a deliberate strategy or a less conscious response style. It is an interesting question whether for the cognitive experimental task data commonly used for the drift diffusion model, high correlations between drift rate and boundary separation are also found.

Our study also has some limitations. First, the number of items used in the study is rather small. Larger sets of items may have allowed us to estimate person-specific variability parameters. Second, the two types of tasks we have investigated are inductive tasks, even though verbal analogies also are knowledge-based. A broader range of tasks would certainly be helpful to test the interpretations we made of our results. Third, the tasks we investigated were of a rather special format, with a fixed and lenient window for responding per item. A more common format would be one with a global window 
for the whole test. Finally, further study would be useful for the variability extensions of the diffusion IRT model. Based on the present study, the modeling approach seems promising, but more and broader applications may provide a more robust underpinning of the approach. 


\section{References}

Akaike, H. (1974). A new look at the statistical model identification. IEEE Transactions on Automatic Control, 19(6), 716-723. doi: 10.1109/TAC.1974.1100705

Bolsinova, M., De Boeck, P., \& Tijmstra, J. (2017). Modelling conditional dependence between response and accuracy. Psychometrika, 82(4), 1126-1148. doi: 10.1007/ s11336-016-9537-6

Bolsinova, M., \& Maris, G. (2016). A test for conditional independence between response time and accuracy. British Journal of Mathematical and Statistical Psychology, 69(1), 62-79. doi: 10.1111/bmsp.12059

Bolsinova, M., \& Molenaar, D. (2018). Modeling nonlinear conditional dependence between response time and accuracy. Frontiers in Psychology, 9(1525), 1-12. doi: 10.3389/fpsyg.2018.01525

Bolsinova, M., \& Molenaar, D. (2019). Nonlinear indicator-level moderation in latent variable models. Multivariate Behavioral Research, 54(1), 62-84. doi: 10.1080/00273171 .2018 .1486174

Bolsinova, M., Tijmstra, J., \& Molenaar, D. (2017). Response moderation models for conditional dependence between response time and response accuracy. British Journal of Mathematical and Statistical Psychology, 70, 257-279. doi: 10.1111/bmsp.12076

Bolsinova, M., Tijmstra, J., Molenaar, D., \& De Boeck, P. (2017). Conditional dependence between response time and accuracy: An overview of its possible sources and directions for distinguishing between them. Frontiers in Psychology, 8, 202. doi: 10.3389/fpsyg.2017.00202

Chen, H., De Boeck, P., Grady, M., Yang, C.-L., \& Waldschmidt, D. (2018a). Curvilinear dependency of response accuracy on response time in cognitive tests. Intelligence, 69, 16 - 23. doi: 10.1016/j.intell.2018.04.001

Chen, H., De Boeck, P., Grady, M., Yang, C., \& Waldschmidt, D. (2018b). A bifactor approach to modeling dependencies between response time and accuracy. Paper 
presented at the annual meeting of the National Council on Measurement in Education (NCME).

De Boeck, P., Chen, H., \& Davison, M. (2017). Spontaneous and imposed speed of cognitive test responses. British Journal of Mathematical and Statistical Psychology, 70(2), 225-237. doi: 10.1111/bmsp.12094

De Boeck, P., \& Jeon, M. (2019). An overview of models for response times and processes in cognitive tests. Frontiers in Psychology, 10, 102. doi: 10.3389/fpsyg.2019.00102

DiTrapani, J., Jeon, M., De Boeck, P., \& Partchev, I. (2016). Attempting to differentiate fast and slow intelligence: Using generalized item response trees to examine the role of speed on intelligence tests. Intelligence, 56, 82-92. doi: 10.1016/j.intell.2016.02.012

Frischkorn, G. T., \& Schubert, A.-L. (2018). Cognitive models in intelligence research: Advantages and recommendations for their application. Journal of Intelligence, 6(3). doi: $10.3390 /$ jintelligence6030034

Gelman, A. (1996). Inference and monitoring convergence. In W. R. Gilks, S. Richardson, \& D. J. Spiegelhalter (Eds.), Markov chain monte carlo in practice (p. 131-143). CRC Press.

Gelman, A. (2006). Prior distributions for variance parameters in hierarchical models (comment on article by Browne and Draper). Bayesian Analysis, 1(3), 515 - 534. doi: 10.1214/06-BA117A

Gelman, A., Carlin, J. B., Stern, H. S., Dunson, D. B., \& A. Vehtari, D. B. R. (2013). Bayesian data analysis (3rd ed.). CRC Press.

Goldhammer, F., Naumann, J., \& Greiff, S. (2015). More is not always better: The relation between item response and item response time in raven's matrices. Journal of Intelligence, 3(1), 21-40. doi: 10.3390/jintelligence3010021

Goldhammer, F., Naumann, J., Stelter, A., Tóth, K., R'olke, H., \& Klieme, E. (2014). The time on task effect in reading and problem solving is moderated by task difficulty and skill: Insights from a computer-based large-scale assessment. Journal of Educa- 
tional Psychology, 106(3), 608-626. doi: 10.1037/a0034716

Hammond, K. R. (1955). Probabilistic functioning and the clinical method. Psychological Review, 62(4), 255 - 262. doi: 10.1037/h0046845

Hoffman, P. J. (1960). The paramorphic representation of clinical judgment. Psychological Bulletin, 57(2), 116 - 131. doi: 10.1037/h0047807

Hornke, L. F. (1999). Benefits from computerized adaptive testing as seen in simulation studies. European Journal of Psychological Assessment, 15(2), 91-98. doi: 10.1027// 1015-5759.15.2.91

Hornke, L. F. (2001). Item generation models for higher order cognitive functions. In S. Irvine \& P. Kyllonen (Eds.), Item generation (p. 159-178). Hillsdale, NJ: Erlbaum.

Hornke, L. F., \& Habon, M. W. (1986). Rule-based item bank construction and evaluation within the linear logistic framework. Applied Psychological Measurement, 10(4), 369380. doi: $10.1177 / 014662168601000405$

Hornke, L. F., \& Rettig, K. (1993). Evaluation und revision einer itembank von analogieaufgaben [evaluation and revision of an item bank of verbal analogy items]. Zeitschrift für Differentielle und Diagnostische Psychologie, 14, 113-128.

Hornke, L. F., \& Wilding, U. (1997). Konstanz von itemparametern bei parallelen itembanken [constancy of item parameters in parallel item banks] (Tech. Rep.). RWTH Aachen University.

Jeon, M., Jin, I. H., Schweinberger, M., \& Baugh, S. (2021). Mapping unobserved itemrespondent interactions: A latent space item response model with interaction map. Psychometrika, 86, 378-403. doi: 10.1007/s11336-021-09762-5

Kang, I., De Boeck, P., \& Ratcliff, R. (accepted for publication). Modeling conditional dependence of response accuracy and response time with the diffusion item response theory model. Psychometrika, Retrieved from https://psyarxiv.com/6jv5n.

Lerche, V., von Krause, M., Voss, A., Frischkorn, G., Schubert, A.-L., \& Hagemann, D. (2020). Diffusion modeling and intelligence: Drift rates show both domain-general 
and domain-specific relations with intelligence. Journal of Experimental Psychology General, 149(12), 2207-2249. doi: 10.1037/xge0000774

Luce, R. D. (1986). Response times: Their role in inferring elementary mental organization. Oxford: Oxford University Publication.

McKoon, G., \& Ratcliff, R. (2012, 04). Aging and iq effects on associative recognition and priming in item recognition. Journal of Memory and Language, 66, 416-437. doi: 10.1016/j.jml.2011.12.001

Meng, X.-B., Tao, J., \& Chang, H.-H. (2015). A conditional joint modeling approach for locally dependent item responses and response times. Journal of Educational Measurement, 52(1), 1-27. doi: 10.1111/jedm.12060

Molenaar, D., \& De Boeck, P. (2018). Response mixture modeling: Accounting for heterogeneity in item characteristics across response times. Psychometrika, 83(2), 279-297.

Molenaar, D., Tuerlinckx, F., \& van der Maas, H. L. J. (2015a). A bivariate generalized linear item response theory modeling framework to the analysis of responses and response times. Multivariate Behavioral Research, 50(1), 56-74. doi: 10.1080/00273171 .2014 .962684

Molenaar, D., Tuerlinckx, F., \& van der Maas, H. L. J. (2015b). Fitting diffusion item response theory models for responses and response times using the r package diffirt. Journal of Statistical Software, 66(4), 1-34. doi: 10.18637/jss.v066.i04

Partchev, I., \& De Boeck, P. (2012). Can fast and slow intelligence be differentiated? Intelligence, 40(1), 23-32. doi: 10.1016/j.intell.2011.11.002

Ratcliff, R. (1978). A theory of memory retrieval. Psychological Review, 85(2), 59-108.

Ratcliff, R. (2002). A diffusion model account of response time and accuracy in a brightness discrimination task: Fitting real data and failing to fit fake but plausible data. Psychological Science, 9(2), 278-291.

Ratcliff, R., \& McKoon, G. (2008). The diffusion decision model: Theory and data for two-choice decision tasks. Neural Computation, 20(4), 873-922. 
Ratcliff, R., Schmiedek, F., \& McKoon, G. (2008). A diffusion model explanation of the worst performance rule for reaction time and iq. Intelligence, 36(1), 10-17. doi: 10 $.1016 /$ j.intell.2006.12.002

Ratcliff, R., Thapar, A., \& McKoon, G. (2009, 12). Individual differences, aging, and iq in two-choice tasks. Cognitive psychology, 60, 127-157. doi: 10.1016/j.cogpsych.2009.09 .001

Ratcliff, R., Thapar, A., \& McKoon, G. (2011). Effects of aging and iq on item and associative memory. Journal of Experimental Psychology: General, 140, 464-487. doi: $10.1037 / \mathrm{a} 0023810$

Ratcliff, R., \& Tuerlinckx, F. (2002). Estimating parameters of the diffusion model: Approaches to dealing with contaminant reaction times and parameter variability. Psychonomic Bulletin \& Review, 9, 438-481.

Schmiedek, F., Oberauer, K., Wilhelm, O., Süß, H.-M., \& Wittmann, W. (2007). Individual differences in components of reaction time distributions and their relations to working memory and intelligence. Journal of experimental psychology. General, 136, 414-429. doi: 10.1037/0096-3445.136.3.414

Schmitz, F., \& Wilhelm, O. (2016). Modeling mental speed: Decomposing response time distributions in elementary cognitive tasks and correlations with working memory capacity and fluid intelligence. Journal of Intelligence, 4(4). doi: 10.3390/ jintelligence 4040013

Schubert, A.-L., \& Frischkorn, G. T. (2020). Neurocognitive psychometrics of intelligence: How measurement advancements unveiled the role of mental speed in intelligence differences. Current Directions in Psychological Science, 29(2), 140-146. doi: 10.1177/ 0963721419896365

Schubert, A.-L., Hagemann, D., Voss, A., Schankin, A., \& Bergmann, K. (2015). Decomposing the relationship between mental speed and mental abilities. Intelligence, 51, 28-46. doi: 10.1016/j.intell.2015.05.002 
Schulz-Zhecheva, Y., Voelkle, M. C., Beauducel, A., Biscaldi, M., \& Klein, C. (2016). Predicting fluid intelligence by components of reaction time distributions from simple choice reaction time tasks. Journal of Intelligence, 4(3). doi: 10.3390/ jintelligence 4030008

Schwarz, G. (1978). Estimating the dimension of a model. The annals of statistics, 6(2), 461-464. doi: $10.2307 / 2958889$

Shaw, A., Elizondo, F., \& Wadlington, P. L. (2020). Reasoning, fast and slow: How noncognitive factors may alter the ability-speed relationship. Intelligence, 83, 101490. doi: 10.1016/j.intell.2020.101490

Spearman, C. (1923). The nature of "intelligence" and the principles of cognition. London: Macmillan.

Spiegelhalter, D. J., Best, N. G., Carlin, B. P., \& Van Der Linde, A. (2002). Bayesian measures of model complexity and fit. Journal of the Royal Statistical Society: Series B (Statistical Methodology), 64(4), 583-639. doi: 10.1111/1467-9868.00353

Sternberg, R. J. (1977a). Component processes in analogical reasoning. Psychological Review, 84(4), 353-378. doi: 10.1037/0033-295X.84.4.353

Sternberg, R. J. (1977b). Intelligence, information processing, and analogical reasoning: The componential analysis of human abilities. Lawrence Erlbaum.

Sternberg, S. (1969). Memory-scanning: Mental processes revealed by reaction-time experiments. American Scientist, 57(4), 421-457.

Tuerlinckx, F., \& De Boeck, P. (2005). Two interpretations of the discrimination parameter. Psychometrika, 70(4), 629-650. doi: 10.1007/s11336-000-0810-3

Tuerlinckx, F., Molenaar, D., \& van der Maas, H. L. J. (2016). Diffusion-based responsetime models. In W. J. van der Linden (Ed.), Handbook of item response theory (p. 283300). Chapman and Hall/CRC.

Turner, B. M., Sederberg, P. B., Brown, S. D., \& Steyvers, M. (2013). A method for efficiently sampling from distributions with correlated dimensions. Psychological Meth- 
ods, 18(3), 368-384.

van der Linden, W. J. (2007). A hierarchical framework for modeling speed and accuracy on test items. Psychometrika, 72(3), 287-308. doi: 10.1007/s11336-006-1478-z

van der Linden, W. J. (2009). Conceptual issues in response-time modeling. Journal of Educational Measurement, 46(3), 247-272. doi: 10.1111/j.1745-3984.2009.00080.x

van der Linden, W. J., \& Glas, C. A. W. (2010). Statistical tests of conditional independence between responses and/or response times on test items. Psychometrika, 75, 120-139. doi: 10.1007/s11336-009-9129-9

van der Maas, H. L. J., Molenaar, D., Maris, G., Kievit, R. A., \& Borsboom, D. (2011). Cognitive psychology meets psychometric theory: On the relation between process models for decision making and latent variable models for individual differences. Psychological Review, 118(2), 339-356. doi: 10.1080/20445911.2011.454498

van Rijn, P. W., \& Ali, U. S. (2017). A comparison of item response models for accuracy and speed of item responses with applications to adaptive testing. British Journal of Mathematical and Statistical Psychology, 70(2), 317-345. doi: 10.1111/bmsp.12101

Wang, C., \& Xu, G. (2015). A mixture hierarchical model for response times and response accuracy. British Journal of Mathematical and Statistical Psychology, 68(3), 456-477. doi: 10.1111/bmsp.12054 


\section{Appendix A: Model Fitting Method}

To fit different versions of the diffusion IRT models to data, we implemented a Bayesian algorithm called Differential Evolution Markov Chain Monte Carlo (DE-MCMC; Turner, Sederberg, Brown, \& Steyvers, 2013) method. This method is known to be robust to a highly-correlated parameter space as that for many process-based models including the diffusion IRT model.

For the Bayesian estimation, prior distributions for the parameters of interest should be specified. For person-wise drift rates and person-wise boundary separations (logtransformed), we used a bivariate normal distribution as the population distribution as similarly done in van der Linden (2007) and Bolsinova, De Boeck, and Tijmstra (2017):

$$
\left(\theta_{p}, \log \left(\gamma_{p}\right)\right) \sim M V N\left(\left[\begin{array}{l}
0 \\
0
\end{array}\right],\left[\begin{array}{cc}
\sigma_{\theta}^{2} & \sigma_{\theta \gamma} \\
\sigma_{\gamma \theta} & \sigma_{\gamma}^{2}
\end{array}\right]\right)
$$

The covariance matrix Equation 2 was given an inverse-Wishart distribution with 4 degrees of freedom and identity matrix as the scale matrix as done in Bolsinova, De Boeck, and Tijmstra (2017). For a person-wise nondecision time, we used a uniform distribution with person-specific ranges: $\tau_{p} \sim U\left(0, \min _{i}\left(T_{p i}\right)\right)$. The lower bound of 0 is because nondecision time should be positive. The person-specific upper bound, which is the personwise minimum RT is because RT is the sum of decision and nondecision times and thus nondecision time should be smaller than all RTs from the person.

For item parameters, we gave $a_{i} \sim U(0,2)$ and $b_{i} \sim N(0,2)$. These distributions were expected to be only weakly-informative, covering sufficiently wide range of parameter values. For example, item time-pressure $a_{i}$ larger than 2 is not empirically expected because it produces a very small decision boundary. This corresponds to very short RTs, which is not appropriate for data from our target ability tests. Also, because we model binary item responses, and the prior population mean of person drift rate is 0 , the effective range for item difficulty $b_{i}$ is not wide (Jeon, Jin, Schweinberger, \& Baugh. 
2021). With prior SD of 2, $1.5 \mathrm{SD}$ (which is 3) leads to the probability (i.e., predicted accuracy) of $\exp (3) /(1+\exp (3))=0.953$ and $2 \mathrm{SD}$ (which is 4$)$ leads to the probability of $\exp (4) /(1+\exp (4))=0.982$. Thus, the current prior specifications would be only weakly-informative and can be overruled by information from data. For variability parameters, whether they were single (apply to all persons and items) or item-specific, we used a half-cauchy(25) prior (Gelman, 2006) for variability in drift rate parameters and a $\operatorname{Beta}(3,2)$ prior (Kang et al. accepted for publication) for variability in starting point parameters.

For each data and each model, we ran 10,000 iterations with 8 chains and discarded the first 2,000 as burn-in. To check if the Bayesian chains converge, we computed the Gelman-Rubin convergence diagnostic $(\hat{R})$ and see if it was smaller than 1.1 for all model parameters (Gelman, 1996; Gelman, Carlin, Stern, Dunson, \& A. Vehtari, 2013). We also obtained posterior densities by chain and visually inspect if they match with each other. When there was a model that has some non-converging parameters, we did not include this model in our model comparison.

\section{Appendix B: Absolute Model Fits}

For the best-fitting model, we conducted posterior predictive checking to assess the absolute model fit (Gelman et al. 2013). To this end, we first randomly chose 1,000 posterior samples of the parameters of the best fitting model. Then, for each of these samples, we generated a random sample of response and RT. This produced 1,000 posterior predictive samples of responses and RTs. Finally, we obtained model predictions of response accuracy and RT distributions (quantiles) with the posterior predictive samples. We contrasted these predictions to the data to check the absolute model fit; provided that predicted values showed good consistency with the data, then we can assure ourselves of a good absolute model fit.

The top three panels in Figure B1 show the posterior predictive checking results of the 

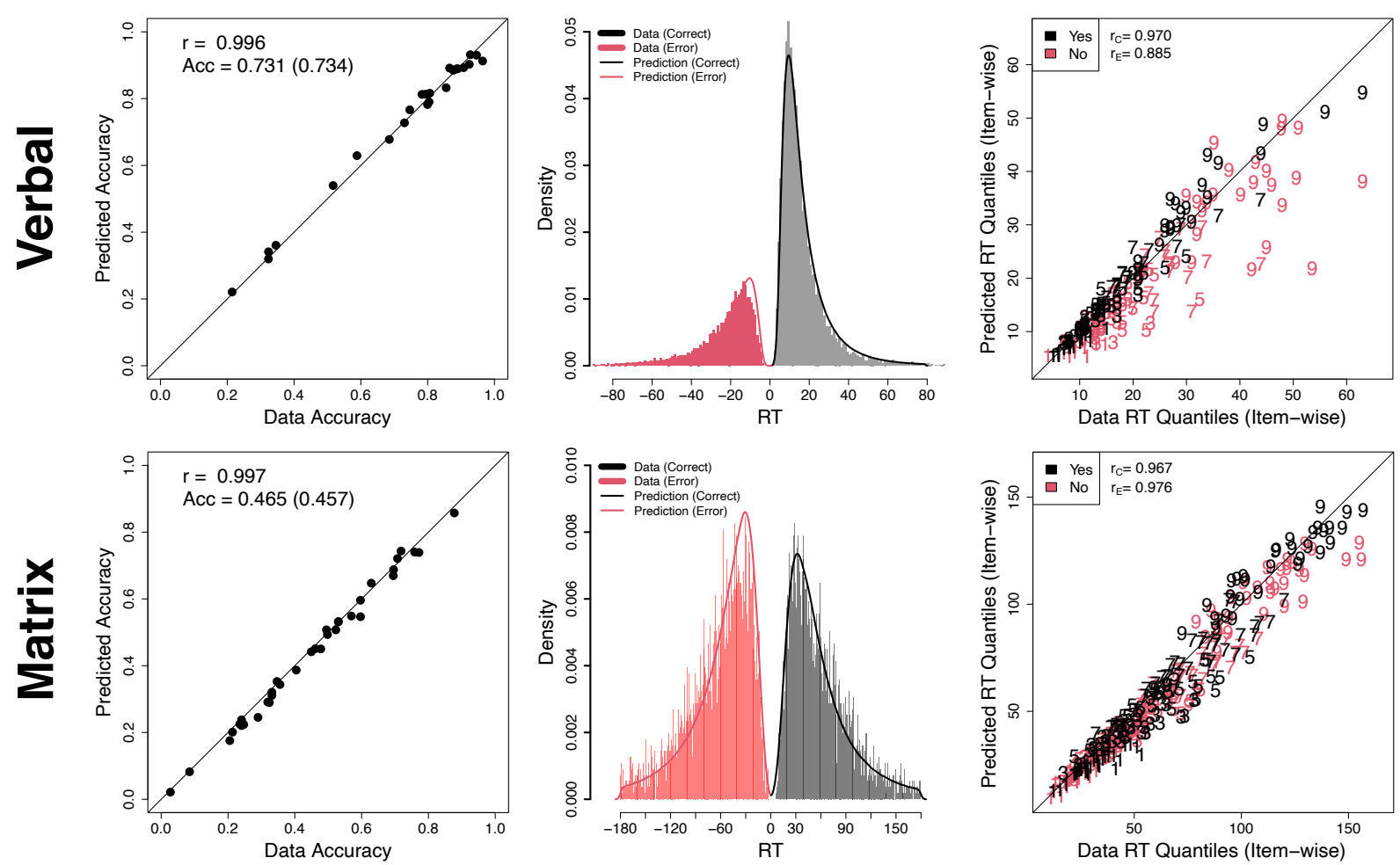

Figure B1: Absolute Model Fits of the Best-fitting Models to Verbal Analogies Data (top) and Matrix Reasoning Data (Bottom): In the left-most panel, item response accuracy values are plotted on the x-axis against the corresponding model predictions on the y-axis. Overall RT distributions (in seconds) are shown in the middle panel. The distribution was obtained by aggregating all persons and all items. The correct RT distribution is indicated by black while the error RT distribution is indicated by red. The histograms show the data distribution and the densities show the model predictions. For visual clarity, the error RTs are scaled negatively and then plotted. The right-most panel shows the item-wise RT quantiles (RT quantiles by items). The data quantiles are plotted on the $x$-axis against the model prediction plotted on the $y$-axis. The numbers $(1,3,5,7$, and 9) represent the 5 quantile points of different items (10\%,30\%, 50\%, 70\%, and 90\% quantiles). The black numbers represent the correct RT quantiles while the red numbers represent the error RT quantiles. ACC: Accuracy. 
verbal analogies data. The best-fitting model was the diffusion IRT model with all itemwise variability parameters in drift rate $\left(\eta_{i}\right)$ and in starting points $\left(s_{z r, i}\right)$ and this model was used to generate posterior predictive samples and model predictions. Item response accuracy is shown in the left-most panel. The accuracy values were obtained by item and plotted on the x-axis against the corresponding model predictions on the $y$-axis. The predictions match the data well with the Pearson correlation of $r=0.996$ as shown at the top-left side of the panel. The overall response accuracy ( 0.731 over all items) is shown below the correlation estimate with the predicted accuracy in parentheses (0.734).

The middle panel shows the overall RT distributions obtained by aggregating all persons and items but separately for correct (black) and error (red) responses. For visual clarity, the error RTs were negatively coded and then plotted. The histograms show the RT distributions of the data while the overlaid densities show the predicted RT distributions. In general, the densities are consistent with the histogram, showing a good model fit. To further inspect the RT predictions, the right panel shows the item-wise RT quantiles (RT quantiles obtained by item). The item-wise RT quantiles calculated from data are plotted on the x-axis against the model predictions on the y-axis. As in the middle panel, RTs for correct responses were colored black and those for error responses are colored red. The numbers $(1,3,5,7$, and 9$)$ represent the 5 quantile points of different items $(10 \%, 30 \%, 50 \%, 70 \%$, and $90 \%$ quantiles). The model predictions show good agreement with data as indicated by the Pearson correlations of $r_{C}=0.970$ for correct responses and $r_{\mathrm{E}}=0.885$ for error responses. The item-wise error RT quantiles deviate more from the diagonal line than the item-wise correct RT quantiles. This is typical in RT data with high or moderately high accuracy because there would be fewer error observations, which makes it more difficult to acquire precise error RT quantiles from data. The overall accuracy of the verbal analogies test is 0.729 , which is not too high, but item-wise accuracy varies a lot in the range of $[0.215,0.964]$. That is, there are some items with only a few error observations and for these items, 1) data error RT quantiles cannot be precisely obtained and 
2) the estimation algorithm depends more on correct responses (and their RTs) than error responses. This will be further discussed below.

The bottom three panels Figure B1 show the posterior predictive checking results of the matrix reasoning data. The best-fitting model was the diffusion IRT model with all item-wise variability parameters in starting points $\left(s_{z r, i}\right)$ but not in drift rate. In general, response accuracy is lower and RTs are longer in the matrix reasoning data than those in the verbal analogies data. The left panel shows that the model predictions on response accuracy are consistent with the data. The overall data accuracy is 0.465 and the predictive value is 0.457 . The predicted item-wise accuracy values match the data well shown as the Pearson correlation of $r=0.997$. The middle and right panels show that the model also performed well in accounting for overall and item-wise RT distributions as shown by the good consistency between the histograms and densities and the Pearson correlations between data and predicted item-wise RT quantiles of $r_{C}=0.967$ for correct responses and $r_{\mathrm{E}}=0.976$ for error responses, with no noticeable bias. Note that, unlike the verbal analogies data, the matrix reasoning data have almost equal proportions of correct and error RTs and thus both distributions were almost equally well captured by the model.

The misfit in the error RT distributions of the verbal analogies data was further investigated with Figure B2. In the left-most panel, the item-wise mean squared errors (MSEs) of error RT quantiles (calculated with five error quantile points per item) are plotted on the $y$-axis against the item-wise data error proportions on the $x$-axis. The plot shows that there are some items with large MSEs and all of them have only a few error observations. In the middle and the right-most panels, the error RT quantiles of the verbal analogies data (used in the top-right panel of Figure B1) are plotted again, but separately by whether item-wise error proportion is greater (middle) or less (right-most) than 0.15. These plots show that error RT quantiles are correctly recovered for the items with error proportion greater than 0.15 , but there are some misfits for the items with fewer error observations. Therefore, the misfit we observed in the top-right panel of Figure B1 can be 

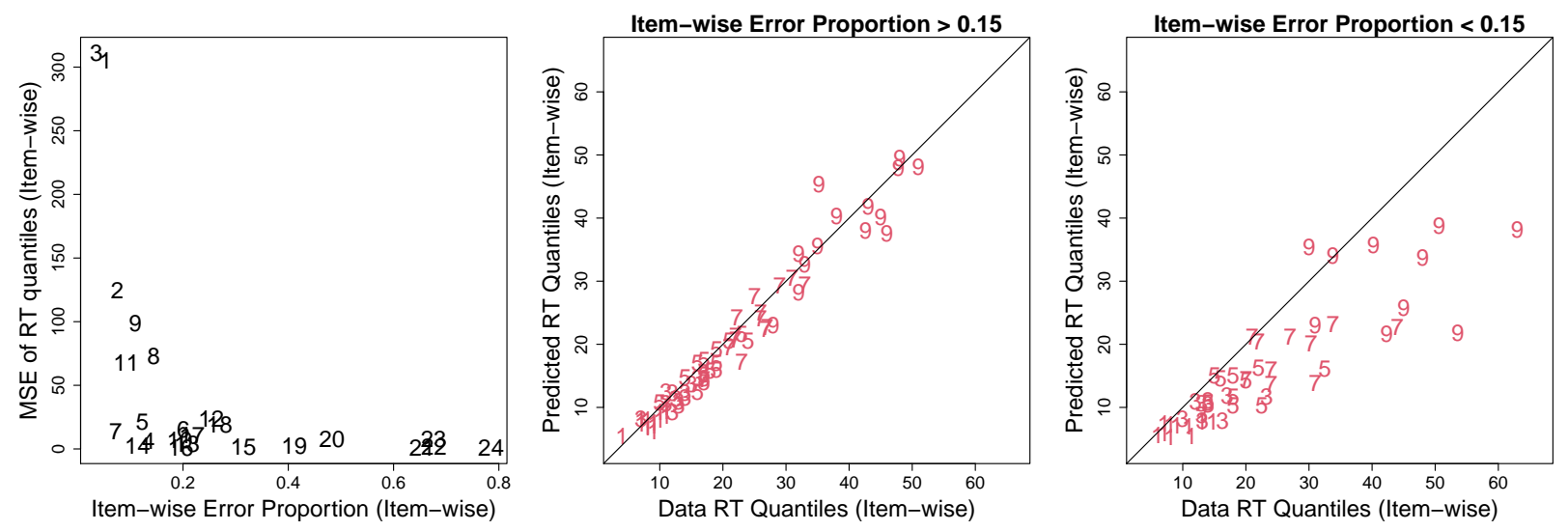

Figure B2: Misfit in the Error RT Distributions of the Verbal Analogies Test. In the leftmost panel, the item-wise mean squared errors (MSEs) of error RT quantiles are plotted on the $y$-axis against the item-wise data error proportions on the $x$-axis. The numbers (from 1 to 24 ) indicate item numbers. In the middle and the right-most panels, the error RT quantiles of the verbal analogies data (used in the top-right panel of Figure B1) are plotted again, but separately by whether item-wise error proportion is greater (middle) or less (right-most) than 0.15 .

attributed to a few number of error observations for items with high accuracy.

\section{Appendix C: Parameter Recovery Simulation}

In this appendix, we present a simulation study to examine if the diffusion IRT models extended with random variability parameters can recover the model parameters reasonably well. Although general parameter recovery of the models was examined in detail in Kang et al. (accepted for publication), the earlier study used a single variability for each of the two random variability components (one in drift rate and the other in starting point) and did not examine item-specific variability parameters we used in the current study. Thus, the simulation study presented here aims to examine this new extension.

We conducted simulations with three conditions that differ by the number of persons $P$ and the number of items $I$. The first simulation was conducted with $P=500$ and $I=20$. Note that the verbal analogies data and the matrix reasoning data we studied in the current study had 904 and 503 persons, respectively, and 24 and 35 items, respectively.

However, the matrix reasoning data had much fewer valid observations (responses 
or person-item pairs) due to a incomplete design; each person responded to a subset of the 35 items (11.1 on average) and each item was responded to by a subset of the 503 persons (159.2 on average). In the second condition, we set $P=500$ and $I=10$, which produced 5,000 pairs of persons and items, and in the third condition, $P=150$ and $I=35$, which produced 5,250 pairs. Note that the number of available persons per item and the number of available item responses per person in the matrix reasoning data would be between those in the second and the third simulation conditions. Also, it can be expected that person parameters would be better estimated with a larger $I$ while item parameters would be better estimated with a larger $P$ despite similar numbers of valid responses.

For each of the conditions, we randomly generated data-generating parameters from the following distributions: $\left(\theta_{p}, \log \left(\gamma_{p}\right)\right) \sim M V N\left(\left[\begin{array}{l}0 \\ 0\end{array}\right],\left[\begin{array}{cc}1 & 0.2 \\ 0.2 & 0.5\end{array}\right]\right), t_{p} \sim T N(2,1 ; 1, \infty)$, $\log \left(a_{i}\right) \sim U(-1.5,0), b_{i} \sim U(-1.5,0.5), s_{z r, i} \sim U(0.15,0.85)$ and $\eta_{i} \sim T N(1,0.5 ; 0.3, \infty)$ where $\operatorname{TN}(\mu, \sigma ; a, b)$ represents a truncated normal distribution with mean $\mu, \operatorname{SD} \sigma$, and range $(a, b)$. Synthetic data of responses and RTs were generated with this set of parameters. Then, we fitted the diffusion IRT model with item-wise random variability parameters to the synthetic data for each of the simulation conditions, with the same prior specifications and estimation method used in our empirical applications (see Appendix A).

There was a convergence issue in our third condition and the full model with both item-wise variability components in drift rate and in starting point did not reach a convergent result. In particular, item-wise drift rate variability parameters $\eta_{i}$ failed to converge. This would be due to the smaller number of persons $(P=150)$ that was not sufficient to constrain this parameter. In contrast, we obtained a convergent result for the full model in the second condition although synthetic data had fewer valid responses in this condition. This would be because we had more persons $(P=500)$ per item and there were only 

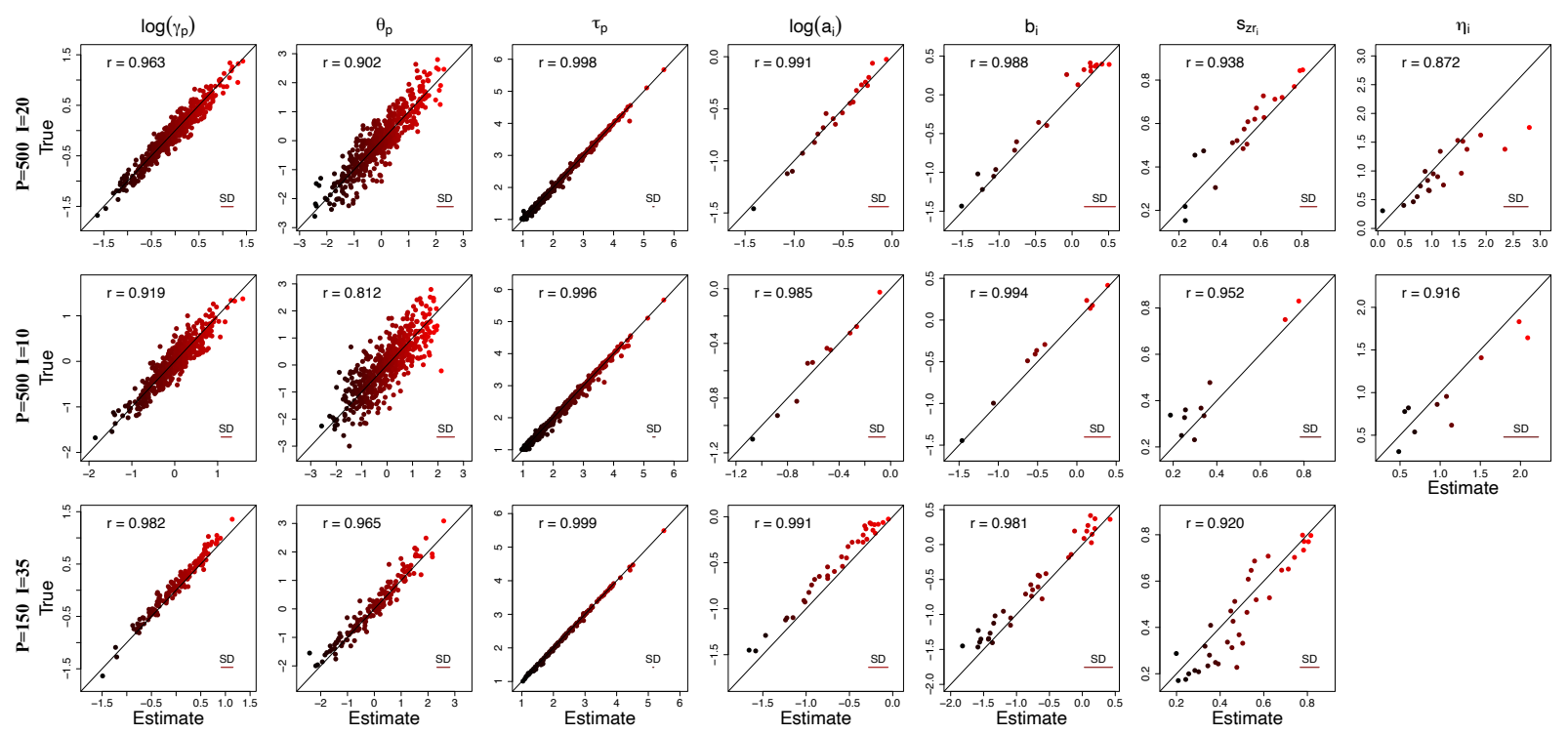

Figure C1: Parameter Recovery of the Diffusion IRT Models with Item-wise Random Variability Parameters. The three rows show the results for the three simulation conditions as denoted on the left, and the columns correspond to the model parameters as denoted on the top. In each panel, the Maximum A Posteriori (MAP) estimate of a parameter is plotted on the $\mathrm{x}$-axis against its true value on the y-axis. At the top-left side of each panel, the Pearson correlations $(r)$ between the MAP estimates and true parameter values are shown. Also, posterior SDs of a parameter are averaged over items (for person-wise parameters) or over persons (for item-wise parameters) and shown at the bottom-right side of each panel as a horizontal bar (i.e., the length of the bar corresponds to the average of posterior SDs). Note that the first two rows show the recovery of the full model with both item-wise variability components in drift rate and in starting point while the last row shows the recovery of the model with item-wise variability in starting point only (no variability in drift rate) because of the non-convergence of the full model.

$I=10$ item-wise parameters to estimate. Note that we ran into a similar convergence issue for the full model fitted to the matrix reasoning data. We interpreted this as due to the small number of respondents available for each item, which is now supported by the non-convergent result in our simulation study. For the third simulation condition, we further examined the $\left(s_{z r, i}\right)$ model (i.e., the diffusion IRT model with item-wise variability in starting point but without variability in drift rate), which was the best-fitting convergent model for the matrix reasoning data. We generated synthetic data with this model (with $\eta_{i}=0$ for all items), fitted the model to the data, and obtained a convergent result.

Figure C1 shows the parameter recovery result. The three rows show the results for 
the three simulation conditions as denoted on the left, and the columns correspond to the model parameters as denoted on the top. Because we examined the recovery of the model with item-wise variability in starting point only (no variability in drift rate) in the third condition, there is a missing panel on the third row. In each panel, the Maximum A Posteriori (MAP) estimate of a parameter is plotted on the $\mathrm{x}$-axis against its true value on the y-axis. At the top-left side of each panel, the Pearson correlations $(r)$ between the MAP estimates and true parameter values are shown. Also, posterior SDs of a parameter are averaged over items (for person-wise parameters) or over persons (for item-wise parameters) and shown at the bottom-right side of each panel as a horizontal bar (i.e., the length of the bar corresponds to the average of posterior SDs). The figure shows that, for all simulation conditions and for all parameters, the MAP estimates are consistent with their parameter values without large bias. Therefore, it can be concluded that the models can recover their parameters reasonably well, including item-wise variability parameters. 